\title{
Designing an intervention strategy for public-interest goods: The California electric vehicle market case ${ }^{\text {iz }}$
}

\author{
Ece Zeliha Demirci*, Nesim K. Erkip \\ Department of Industrial Engineering, Bilkent University, Ankara, Turkey
}

\section{A R T I C L E I N F O}

Article history:

Received 18 December 2014

Accepted 1 August 2016

Available online 10 August 2016

Keywords:

Newsboy problem

Case study

\begin{abstract}
A B S T R A C T
We study the intervention problem for public-interest goods. Public-interest goods are known as goods with positive externalities, allowing the consumer as well as others who do not pay for them benefit from the consumption. Health related goods, such as vaccines, or products with less carbon emissions are well known examples. We consider a supply chain for such a product. Generally, wider adoption or usage of such goods is ensured by the intervention of a central authority in their supply chain. We explore the problem for a setting composed of a retailer and a central authority. The main goal of the central authority is to design and fund an intervention scheme so that decisions of the channel are in line with the good of society, specified as a social welfare function. We propose two intervention tools applied simultaneously: (1) investing in demand-increasing strategies, which affects the level of the stochastic demand in the market; and (2) rebates that affect revenue per unit received by the retailer. We introduce a model that determines a utility maximizing intervention scheme and further investigate the model. We also present two decentralized approaches as benchmarks. Finally, we conduct a case study for California's electric vehicle market and validate our findings by a detailed analysis of the results, including comparisons with the current practice.
\end{abstract}

(c) 2016 Elsevier Ltd. All rights reserved.

\section{Introduction}

This study is motivated by public-interest goods, which can also be referred as goods with positive externalities. Health related products, energy efficient appliances, and recent developments in green technology (e.g., electric vehicles, solar panels, etc.) are some notable examples. A distinguishing property of these types of goods is that in addition to consumers, third parties (who do not pay for them) enjoy the benefits of their consumption. For instance, people who get the influenza vaccination reduce the chances of non-vaccinated people getting the flu. As the examples indicate, the usage of public-interest goods tremendously benefits individual customers and the majority of the society, so a central authority usually intervenes in the supply chain for the good of the system. Significant examples of intervention include the intervention of the US government, the World Bank, the Global Fund to Fight AIDS, and Tuberculosis and Malaria in distributing medicines, vaccines, and fortified foods to countries in need [33]; the intervention of the German government in the solar panel market [25]; the intervention of the French government in conjunction

\footnotetext{
This manuscript was processed by Associate Editor Bish.

* Corresponding author.

E-mail address: edemirci@bilkent.edu.tr (E.Z. Demirci).
}

with the European Union in the petro-chemical industry to encourage biofuel production [4]; and the intervention of the US government and states in the electric vehicle market [37]. The ultimate role of a central authority in such cases is to design and fund an intervention scheme that will enable the chain to choose decisions for the benefit of the society, social welfare. The key question, then, is how to design an intervention scheme that maximizes social welfare.

This paper considers a general setting composed of a retailer and a central authority that regulates the system through an intervention scheme. The intervention has the goal of maximizing expected utility (social welfare) rather than only maximizing expected profit. A critical consideration in designing an intervention mechanism is how it should be incorporated into the system. One alternative is to invest in demand-increasing strategies, such as advertising, education, research and development, and awareness campaigns, so that the demand pool is enhanced in the medium to long run. Another alternative can be either offering rebates to customers or administering subsidies to each unit sold. In fact, rebates and subsidies per unit sold operate similar to each other in terms of improving availability of the product at the retailer via increased unit revenue, and at the same time making the good more attractive for the customers given the level of their willingness to pay. So, we refer to the second intervention tool as rebates in the rest of the paper. Analysis of interventions through 
subsidies or rebates is common in the literature, especially for public-interest goods. For example, Raz and Ovchinnikov [30] consider an intervention mechanism consisting of rebates and subsidies for a general class of public-interest goods; Mamani et al. [27] consider a subsidy program to achieve optimal vaccination coverage, and Lobel and Perakis [25] consider subsidies to achieve a desired adoption target for solar photovoltaic technology. Our work is the first to consider alternative strategies for intervention, affecting both supply and demand. Specifically, we propose a joint mechanism, where the central authority uses investment in demand-increasing strategies and rebates simultaneously. In some sense, then, the problem is to decide on the optimal allocation of the central authority's budget among these two intervention tools.

We formulate this framework via bilevel programming. A bilevel programming problem is a hierarchical optimization problem that includes two mathematical problems within a single instance, one of which is part of the constraints of the other. To summarize, an upper-level decision maker or leader makes his optimal choice first and then a lower-level decision maker or follower makes his decision by optimizing his objective given the dominant player's action. A distinguishing property of this programming is that each player's decision is affected by the other's decision, but is not completely controlled by it (see [10,3] for more details). In our case, the central authority is the leader, whose objective is to maximize social welfare, whereas the retailer is the follower, whose objective is to maximize expected profit. The central authority decides on the direct investment amount for the demand-increasing strategies and on the rebate amount per unit, then the retailer decides on the order quantity. There are few welldocumented cases of direct investment response functions, but they are generally assumed to be concave for advertising (e.g. $[19,22,1,23])$, and we follow the same assumption in this paper. The concave response function indicates that as the money invested increases, so does the expected demand, but with a monotonically diminishing rate. On the other hand, the retailer's problem is a newsvendor problem incorporating the rebate amount. The retailer decides on the profit maximizing order quantity. In addition to developing the modeling perspective, we characterize the optimal intervention strategy and provide useful insights for regulating these goods. We also present three benchmark approaches: one is a no-intervention case and two are decentralized approaches that work with a predetermined rebate amount. Finally, we provide a detailed case study for the California electric vehicle market that assesses the performance of our approach relative to the current mechanism and the applicability of the proposed model.

The first contribution of this paper is using bilevel programming for modeling a newsvendor environment with welfare implications. Designing a joint mechanism consisting of investment in demand-increasing strategies as an additional intervention tool and considering a budget are also novel. Further, we introduce several variations of the model as benchmarks. Finally, we conduct a case study to analyze the benefit of using the proposed mechanism by implementing a novel parameter calibration approach.

The remainder of the paper is organized as follows. Section 2 summarizes the related work in the literature, Section 3 presents our model, along with highlights of some analytical results, Section 4 introduces benchmark approaches, Section 5 proceeds with a case study and computational results, and finally, Section 6 presents concluding remarks.

\section{Literature review}

This paper considers the supply chain of a public-interest good, hence it is closely related to the literature on economics and operations management.

The economics literature has focused on policy design for regulating monopolies in the public-interest (see [17,34] for further details). Subsidy, tax, and lump-sum transfers are frequently used as public policy instruments in welfare economics. The details on how they are used and their effects on the economy are discussed thoroughly in [16]. Moreover, the impact of intervention tools (such as subsidies and advertising) for accelerating the diffusion of a new product is investigated in the literature. In this context, government is concerned with maximizing the number of adopters while determining the intervention scheme. Here, the intervention tools directly affect the adoption level. On the other hand, in our paper we study the intervention problem in a newsvendor setting, thus the tools are affecting the order quantity. Some examples from this stream of literature are: $[14,18,11]$. Horsky and Simon [14] examine the effect of firm's advertising strategy on the adoption level of a new product, whereas Kalish and Lilien [18] study the problem of determining a time dependent subsidy scheme under a predetermined government budget In a recent study, De Cesare and Di Liddo [11], innovation diffusion problem is examined for a Stackelberg game. The government chooses the subsidy amount given a predetermined budget, whereas the monopolist producer determines the pricing and advertising strategies.

There is a stream of studies that deals with incentive mechanism and multi-agent decision making for hierarchical systems in the context of operations. In hierarchical systems such as supply chains, health care systems or service organizations, each stage attempts to maximize its own profit, which nevertheless may not coincide with the optimal decisions for the entire system. Two early examples of such studies are Schneewei $\beta$ [31] and Schneeweiss [32]. The latter is a review of more general systems, where decisions are taken in a distributed manner under available information for each agent. Additionally, such systems can be analyzed under varying decision time scales and asymmetric information availability of agents $[39,40]$. Compared to this stream of literature, our approach considers a central authority with all available information.

When we look at the supply chain literature, we see an extensive literature focusing on the impact of incentives on the echelons in a newsvendor environment. Instead of welfare/utility implications, they focus on the profits of firms while designing contracts. (See [5] for a detailed review of the supply chain contracting literature.) Although the problem under consideration is a typical problem seen in various settings, studies that combine an intervention mechanism and social welfare in a newsvendor setting are scarce and generally focus on a certain product (usually vaccines) and consider subsidies or rebates as intervention tools.

Refs. [8,12,27,2] are some examples that analyze welfare implications in the vaccine market while determining the optimal coverage level. All these studies consider specific details of the vaccine market, such as infection dynamics and/or yield uncertainty. Chick et al. [8] assume that demand is exogenous to their model, whereas Deo and Corbett [12] assume that the price is set after yield is realized. However, Arifoğlu et al. [2] and Mamani et al. [27] incorporate consumer behavior in this context. The features of the above-mentioned studies make them inapplicable to other types of public-interest goods. Regarding health related products, Taylor and Xiao [33] study design of subsidies from the perspective of a donor with a budget constraint for improving the availability of malaria drugs. The authors show that the optimal subsidy scheme of donor should include only purchase subsidy 
(i.e. optimal sales subsidy should be zero). Zhang et al. [40] is an application of the multiscale decision theory approach to health care. Similar to our approach, objective functions of stakeholders are monetized.

Lately, the operations management literature has been dealing with implications of intervention mechanisms on sustainability (e.g., [24]) and green technologies (e.g., [21,25,15]). Liu et al. [24] assess the effects of government incentives on the recycling of waste electrical and electronic equipment in a dual channel setting. Our paper is mostly related to studies on promoting green technologies. In this domain, Krass et al. [21] investigate the effects of fixed cost subsidies and taxes placed on emissions regarding the adoption of green technologies. The authors consider a deterministic price dependent demand and a joint mechanism composed of taxes and fixed cost subsidies for regulation purposes. Lobel and Perakis [25] propose a subsidy scheme for motivating the adoption of solar panels. Instead of maximizing the social benefits of this technology, they use an adoption target level for deriving the optimal policy. Huang et al. [15] analyze the impact of a government's subsidy incentive scheme for promoting electric automobiles. Kim et al. [20], on the other hand consider a related operational model for electric automobiles and conclude that operational arrangements will be instrumental in increasing the demand for the product; this can be considered as an example for demand-increasing strategies.

Raz and Ovchinnikov [30] and Cohen et al. [9] are closest to our work in terms of analyzing welfare implications in a newsvendor environment. Our newsvendor setting differs from these studies in that they present a price-setting newsvendor model, whereas we assume that demand distribution changes based on investment in demand-increasing strategies. The former consider a joint intervention mechanism composed of subsidies and rebates for improving the availability of the public-interest good and maximizing social welfare. Also, they use a specific form of social welfare function consisting of a firm's profit, consumer surplus, an externality benefit, and government costs. Cohen et al. [9] study a subsidy design problem for early stages of green technology adoption. Their model finds a subsidy scheme that maximizes welfare (composed of the supplier's profit, a consumer surplus, and government expenditures) in addition to satisfying the given adoption target level. Both studies enrich their findings by a case study for Chevy Volt in the US market.

Our paper differs from the above-mentioned studies in three dimensions: (1) a joint intervention mechanism, consisting of demand-increasing strategies and rebates; (2) an explicit budget consideration on the joint intervention mechanism; and (3) a relatively general social welfare function with the emphasis on optimizing the budget allocation problem.

\section{Model}

This section describes a unifying framework to design an intervention mechanism for a general class of public-interest goods. We utilize this framework for characterizing a strategy that will encourage the usage of this type of good.

We study the problem in a setting composed of a retailer and a central authority. In this context, the retailer refers to either an entity that both manufactures and sells the good, or a coordinated manufacturer-retailer system. The retailer sells the good to individual customers and by its consumption not only the buying individual but also other individuals benefit. The main goal of the central authority in this framework is to regulate the system to increase the good's availability and consumption, and hence collective societal welfare.
The central authority regulates the system through a joint intervention mechanism composed of two different tools: (1) investment in demand-increasing strategies; and (2) rebates. The central authority decides on the direct investment amount made in demand-increasing strategies $B_{d}$, the total amount assigned to rebates $B_{r}$, the rebate amount $r$, and the budget $B$. Depending on $B_{d}$, the distribution function of demand changes. Note that $r$ is a rebate available to the end customers. More specifically, let $p$ denote the price the customer is willing to pay for the good; then the retailer earns a revenue of $p+r$ from each unit sold. This scheme helps customers buy a good that costs more money than they would otherwise be willing to pay and improves the availability of the good at the retailer. We define central authority's objective function as $u\left(Q, B_{d}\right)-B$ to maximize utility (or can be named as social welfare), $u\left(Q, B_{d}\right)$ reflecting benefits to society in monetary units, and $B$ capturing the budgetary expenses of intervention mechanism.

We model the retailer's problem similar to a newsvendor problem. The retailer decides on the order quantity $Q$ by incurring a unit acquisition cost $c$, gaining a unit retail price $p+r$, and a unit salvage price $s$ for unsold goods, with the intention of maximizing the expected profit. However, the distribution of demand depends on the amount the central authority invested in demandincreasing strategies, $B_{d}$, with pdf $f_{B_{d}}\left(\right.$.) and $\operatorname{cdf} F_{B_{d}}($.). Throughout the study it is assumed that the cdf of demand is monotone increasing in its argument. The monotonicity of $F_{B_{d}}($.) allows us to use the following implicit fact while showing our results: as the fractile increases, $Q$ also increases. We assume that there is a family of demand distribution functions dependent on $B_{d}$, and that changes in the value of $B_{d}$ lead to a first order stochastic dominance order between cdfs, i.e. let $\overline{B_{d}}>\widehat{B_{d}}$; then $F_{\overline{B_{d}}}$ has a first order stochastic dominance over $F_{\widehat{B_{d}}}$. Specifically, we impose the assumption that the cdf of demand at a given value is a decreasing function of $B_{d}$. Thus, it is guaranteed that as $B_{d}$ increases, so does $Q$.

The bilevel programming formulation of the problem is as follows:

Model JM : $\max _{r, B_{d}, B_{r}, B} u\left(Q, B_{d}\right)-B$

s.t. $\quad B_{d}+B_{r} \leq B$

$r \mathbb{E}\left[\min \{Q, D\} \mid B_{d}\right] \leq B_{r}$

$r \geq c-p$

$r \geq 0$

$B_{d}, B_{r} \geq 0$

$\max _{Q} \mathbb{E}\left[P(Q) \mid B_{d}\right]$

where $\mathbb{E}\left[P(Q) \mid B_{d}\right]=\int_{0}^{Q}((p+r) x+s(Q-x)-c Q) f_{B_{d}}(x) \mathrm{d} x+\int_{Q}^{\infty}(p+r-$ c) $Q f_{B_{d}}(x) \mathrm{d} x$ is the retailer's expected profit.

Notice that $D$ denotes the demand. Eqs. (1)-(6) express the upper-level problem while (7) corresponds to the lower-level problem. Constraint (2) ensures that the money invested in $B_{d}$ and $B_{r}$ is not more than the budget, whereas constraint (3) limits the expected total rebate amount to the budget allocated to rebates. Note that $B_{d}$ indicates the level of demand to be considered. Constraint (4) restricts the values that $r$ can take, i.e. it assures that $r$ is greater than $c-p$ so that the cost of underage is greater than 0 . This constraint assures a minimum rebate needed to have the product available in the market. The non-negativity of $r, B_{d}$, and $B_{r}$ are guaranteed by (5) and (6). Last, (7) reflects the retailer's problem.

The context discussed can be viewed as a classical leaderfollower game, in which the central authority has the role of leader 
(or a higher-level decision maker), with the objective of maximizing utility (1) and the retailer has the role of follower (or a lower-level decision maker), with the objective of maximizing expected profit (7). Note that by modeling this framework by bilevel programming, we do not solely maximize utility or expected profit; rather we maximize the retailer's expected profit under the dominant objective of the central authority.

\section{Remark 1.}

(i) The total budget will be summation of $B_{d}$ and $B_{r}$ at the optimal solution. Thus, $B$ can be eliminated from the formulation using equality.

(ii) $r \mathbb{E}\left[\min \{Q, D\} \mid B_{d}\right]=B_{r}$ holds at the optimal solution.

Remark 2. For a given $B_{d}, B_{r}$, and $r, \mathbb{E}[P(Q)]$ is concave in $\mathrm{Q}$ thus the solution for the lower-level optimization problem is unique, implying that the rational reaction set $R\left(B_{d}, B_{r}, r\right)$ is single valued and unique. The uniqueness of $R\left(B_{d}, B_{r}, r\right)$ guarantees that the leader achieves his maximum objective (by Proposition 8.1.1, p. 303 of [3]).

Lemma 3.1. $\mathbb{E}[P(Q)]$ is concave in $Q$ for every $B_{d}, B_{r}$, and $r$. This assures that the solution to the lower-level problem is unique, implying that it can be replaced by its first-order condition (by p. 308 of [3]).

Accordingly, Model JM can be written as the following singlelevel mathematical program:

Model JM-SLP : $\quad \max _{r, B_{d}, B_{r}, Q} u\left(Q, B_{d}\right)-\left(B_{d}+B_{r}\right)$

s.t. $\quad r \mathbb{E}\left[\min \{Q, D\} \mid B_{d}\right]=B_{r}$

$r \geq c-p$

$r \geq 0$

$B_{d}, B_{r} \geq 0$

$F_{B_{d}}(Q)=\frac{p+r-c}{p+r-s}$

A general bilevel programming problem is the most challenging of bilevel programs both theoretically and computationally. By reducing the Model JM to a single-level program, we obtain a relatively easy program to solve. Henceforth, we continue our analysis with Model JM-SLP, which is a standard nonlinear program with a nonlinear objective function and constraints. Note that the constraint set of Model JM-SLP is not necessarily convex.

\subsection{Special case: societal benefit is a linear function of expected sales}

In this subsection, we analyze Model JM-SLP for a specific objective function of the central authority and mean demand function.

Assumption 1. Suppose that utility function is defined as $\beta \mathbb{E}\left[\min \{Q, D\} \mid B_{d}\right]-\left(B_{d}+B_{r}\right)$. Note that $\beta$ times expected sales is used to capture the monetary benefits to society, where $\beta$ is the monetary value (\$) per expected sales. In practice, the central authority is generally interested in increasing the number of adopters, so expected sales is a reasonable measure to quantify benefits.

Assumption 2. Similar to the approach in the advertising literature, we consider an increasing and concave response function of
$B_{d}$, which is in the following form:

$\mu\left(B_{d}\right)=\mu_{\infty}-\frac{d}{\left(1+k B_{d}\right)^{a}}, \quad$ where $a, d, k>0$.

Specifically, mean demand increases as $B_{d}$ increases, but with a monotonically diminishing rate. In this form, as $B_{d}$ approaches infinity, demand distribution approaches a limiting distribution with a mean of $\mu_{\infty}$. Note that if other parameters of a distribution are also affected by $B_{d}$, they can be modeled similar to (14).

Under this utility function, we can modify the model Model JMSLP as follows:

Model SC : $\max _{r, B_{d}, Q}(\beta-r) \mathbb{E}\left[\min \{Q, D\} \mid B_{d}\right]-B_{d}$

s.t. $\quad F_{B_{d}}(Q)=\frac{p+r-c}{p+r-s}$

$r \geq c-p$

$r \geq 0$

$B_{d} \geq 0$

We further analyze Model SC under specific distributions for demand. We consider exponential and lognormal distributions to represent family of distributions induced by $B_{d}$.

Corollary 3.1. Consider the Model SC given in (15)-(19). Demand follows a family of distributions dependent on $B_{d}$, where mean of the distribution follows (14). If the family of demand distributions is exponential or lognormal with constant coefficient of variation, then the optimal rebate amount is independent of optimal $B_{d}$.

Proof is presented in Appendix A.

Corollary 3.1 indicates that the optimal rebate amount is not affected from investment made in demand-increasing strategies, efficiency of the strategies, and mean demand before and after investment. From another perspective, the rebates are independent of the planning horizon considered, as one would think that planning horizon considered will change the parameters of demand. From the perspective of retailer, optimal fractile value is constant, regardless the demand parameters, as dictated by the form of the objective function.

Note that for the lognormal distribution, we hold the coefficient of variation constant with respect to the changes in the values of $B_{d}$, i.e. variation of demand increases with the increase in mean demand. Next, we consider an environment in which demand-increasing strategies will also reduce variability in the following form:

$c v\left(B_{d}\right)=c v_{\min }+\frac{c v_{i m p}}{\left(1+k B_{d}\right)^{a}}, \quad$ where $a, k>0$.

Note that we can define $c v_{i m p}$ as portion of the coefficient of variation value that can be eliminated. In particular, coefficient of variation decreases as $B_{d}$ increases, but with a monotonically diminishing rate. As $B_{d}$ approaches infinity, the demand distribution approaches a limiting distribution with a mean of $\mu_{\infty}$ and coefficient of variation of $c v_{\text {min }}$.

Corollary 3.2. Consider the model Model SC given in (15)-(19), mean demand function given in (14), and coefficient of variation function given in (20). If demand is lognormally distributed, then the optimal rebate amount will be dependent on the optimal $B_{d}$.

Proof is presented in Appendix A.

Intuitively, if demand increasing strategies also decrease the variability of demand, the optimal rebate amount, i.e. optimal fractile, becomes a function of $B_{d}$, as well. 


\section{Benchmark approaches}

This section explores three benchmark cases that can be used to assess the performance of our approach. The benchmark approaches considered are representations of the mechanism taking place in practice, where the rebate amount is decided by the central authority [7].

The first benchmark is an obvious one, no-intervention case, which may help us better understand the impact of intervention in this type of system. However, if $c>p$, the system would not operate without intervention. Hence, we cannot employ a no intervention scenario even as a benchmark for $c>p$ cases.

The second benchmark is a decentralized approach, which is explained in detail in the next subsection, and the third benchmark emerges as an extension of it. Note that Remark 1 applies to these benchmarks as well.

\subsection{Decentralized approach}

The difference of the decentralized approach from the joint mechanism is that the problem is solved for a prespecified rebate amount. In other words, decisions about intervention tools are not made jointly; specifically, the rebate amount is preset by the central authority. Under certain demand functions considered in Corollary 3.1, the preset amount by the central authority may be the optimal solution. However, under conditions stated by Corollary 3.2, the rebate amount is a function of $B_{d}$.

Our model formulation for this approach is as follows:

Model DA : $\max _{B_{d}, B_{r}, Q} u\left(Q, B_{d}\right)-\left(B_{d}+B_{r}\right)$

(9), (12), (13)

\subsection{Base decentralized approach}

Inspired from the previous case, we construct another reference, in which the central authority regulates the system only by rebates that are predetermined by the central authority (i.e. $B_{d}=0$ in this case). We call this approach the base decentralized approach henceforth. The aim of this approach is to see how the system operates with a preconcerted rebate amount. No optimization is needed; newsvendor quantity and expected sales corresponding to the predetermined rebate amount are computed, and central authority will assign $B_{r}$ accordingly. The following lines summarize Model BDA:

Model BDA : Computeu( $\left.Q, B_{d}=0\right)-B_{r}$

$$
\begin{aligned}
& \text { where } F_{\left(B_{d}=0\right)}(Q)=\frac{p+r-c}{p+r-s} \\
& r \mathbb{E}\left[\min \{Q, D\} \mid B_{d}=0\right]=B_{r}
\end{aligned}
$$

\section{Case study: the California electric vehicle market}

In this section, we employ the joint intervention mechanism introduced in Section 3 to explore the intervention mechanism in the California electric vehicle (EV) market.

According to a report of the World Business Council for Sustainable Development [38], the global light duty vehicle fleet is projected to be two billion by 2050 . Globally, light duty vehicles are the main contributor of greenhouse gas emissions and depletion of fossil fuel resources [13]. As a response to the increasing pattern in vehicles on the road and its associated implications on climate change and resources, EVs have received increased attention from environmentalists, industry, governments, and academics in the last decade, and have emerged as a strong alternative to conventional internal combustion engine vehicles. As EVs are considered a significant technological breakthrough with potential environmental benefits, they can be categorized as public-interest goods. However, they face a combination of cost and performance issues that limit their competitiveness, which is why their introduction in the market is ensured by governmental policies. Foremost examples of such policies are in the US and European Union, which promote EVs through several programmes and strategies. A notable number of governments have also announced the number of EVs they aim to have on roads by certain dates. For instance, in 2011, US President Obama expressed a target of one million EVs on the road by 2015.

In this study, we consider the intervention design problem for a specific application, the California EV market. Note that we refer to plug-in hybrids (PHEVs) and all electric vehicles (also called battery-electric vehicles (BEVs)) as EVs throughout the paper. In the US, various subsidy schemes have been adopted at the federal level and even the local level to encourage EV sales. Under the current policy at the federal level, the government offers subsidies in the form of a federal income tax credit of up to $\$ 7,500$ for EVs purchased in or after 2010, based on the capacity of the battery used to fuel the vehicle. ${ }^{2}$ At the state level, California implements a special consumer incentive program, the Clean Vehicle Rebate Project (CVRP), to promote the adoption of clean vehicles. ${ }^{3}$ The project was launched in March 2010 and expected to end in 2015. Under the CVRP, individuals, nonprofits, government entities, and businesses can receive a rebate up to $\$ 5000$ on top of the $\$ 7500$ federal tax credit for the purchase of eligible vehicles, which include zero emission vehicles (ZEVs) (BEVs are categorized as ZEVs), PHEVs, neighborhood electric vehicles (NEVs), and zero emission motorcycles (ZEMs). According to current statistics, California is the leading state in clean vehicle adoption: although the state constitutes $10 \%$ of the total US car market, $40 \%$ of all PHEVs purchases are from California. ${ }^{4}$ Also, a May 2013 PHEV driver survey ${ }^{5}$ reports that $47 \%$ of purchasers rate the state rebate as the most important factor in their decision to purchase a PHEV. From these statistics, we can better understand the significance of a state rebate on the motivation for EV purchases.

Most of the infrastructural investment to increase the efficiency and reliability of the technology is expected to attract more customer demand (see p. 8, [36]). Following the discussion on the pp. 6-7 of the document [36], on the other hand, these R\&D investments mostly were completed before 2012, and hence we assume that those effects are already reflected in the current demand estimates.

Unfortunately, however, the statistics indicate that current policies are insufficient to reach nationwide goals on clean vehicle market growth. According to an Information Technology and Innovation Foundation report [13], the right policy to encourage mass adoption of electric vehicles is a combination of subsidies and battery research funding to remove technological barriers, which parallels the mechanism we introduce in Section 3. Electric vehicles have been only recently introduced in the market and their ongoing development is open ended, dependent on investments in battery improvement technologies and production

\footnotetext{
${ }^{1}$ http://www.whitehouse.gov/the-press-office/2011/01/25/remarks-presidentstate-union-address.

${ }^{2}$ http://www.fueleconomy.gov/feg/taxevb.shtml.

3 http://energycenter.org/clean-vehicle-rebate-project.

4 http://www.government-fleet.com/channel/green-fleet/news/story/2013/03/ california-ev-and-zero-emission-rebate-program-extended.aspx? prestitial =1.

${ }^{5}$ http://energycenter.org/clean-vehicle-rebate-project/vehicle-owner-survey/ may-2013-survey.
} 
processes. Several research projects are currently underway to improve battery technology, but these developments must be fostered more aggressively. Thus, balancing available funds between research and development, and rebates is a key issue in resolving the adoption problem.

We use the newsvendor context in the EV example, which implies that there is a single period and a monopolist retailer. Obviously, the retailer does not make a single production decision; instead he is making major decisions each year, and then more detailed decisions each month or week. Hence, it is critical to emphasize that the quantities found should be interpreted as capacity decisions of the retailer. Also, there are many EV models and hence competing manufacturing firms available in the market. However, in this problem we aggregate the retailers and investigate the industry-wide problem, because our main concern is the reaction of the industry to the intervention mechanism.

Specifically, our numerical study addresses the following issues: (1) the value of the joint mechanism, (2) the performance of the joint mechanism in short-term versus long-term planning, (3) the impact of uncertainty on the results, and (4) the applicability of the proposed model.

Before proceeding with the numerical results, we first describe the demand model, objective function, and how we choose and calibrated parameter values.

\subsection{Demand model and objective function}

We use two different distributions to represent EV demand and conduct separate computational studies to evaluate the robustness of the results with respect to distribution. The demand of a product like an EV, which is in the early stages of adoption, is expected to be highly uncertain. So, at first we assume demand to be exponentially distributed. Subsequently, we consider the case that demand follows a lognormal distribution, as it allows us to assess the impact of demand uncertainty on the results. Note that we consider the mean demand function given in (14) for both distributions.

Motivated by the EV market's circumstances, we choose the central authority's utility function as given in Assumption 1. By utilizing this objective, we choose expected sales value to assess the benefits to society for this application. The US government is concerned with increasing the number of EVs on the road, and President Obama has announced an adoption target. Therefore, the expected sales will be a realistic function to capture benefits to society and it is consistent with the current market environment. ${ }^{6}$

\subsection{Choice of parameter values}

The cost parameters in this numerical study are based on the market data of a specific model, the Nissan Leaf. The Leaf is an all electric vehicle constituting a global market share of $45 \%$ as of the first quarter of 2014 [28]. The manufacturer's suggested retail price (MSRP) for the Leaf 2013 is $\$ 28,800$ [29] in the US, and it is eligible for a rebate of $\$ 2500$ by the $\mathrm{CVRP}^{7}$ on top of a federal income tax credit of $\$ 7500 .^{8}$ Thus, the price the customer is willing to pay is reduced to $\$ 18,800$. Also, from market data we find out that the factory invoice, i.e. the amount the manufacturer charges the dealer (c), is $\$ 26,986$ [35].

\footnotetext{
${ }^{6}$ One discussion is whether increasing sales of vehicles really does benefit society, regardless of whether what is sold is an EV. The utility function considered is expected to decrease for some large sales levels due to both concavity of expected sales and subtraction of budgetary expenses from monetary value of expected sales.

7 http://energycenter.org/clean-vehicle-rebate-project.

${ }^{8}$ http://www.fueleconomy.gov/feg/taxevb.shtml.
}

Table 1

Demand forecasts of EVs.

\begin{tabular}{lcc}
\hline Period & Low Scenario & High Scenario \\
\hline $2011-2013$ & 43,113 & 332,559 \\
$2011-2015$ & 108,907 & $1,081,703$ \\
$2011-2022$ & 834,489 & $3,574,670$ \\
\hline
\end{tabular}

Table 2

Demand model's parameters for base case scenario.

\begin{tabular}{lllrrr}
\hline Distribution & cv & \multicolumn{1}{l}{$s$} & $\mu_{\infty}-d$ & \multicolumn{1}{c}{$d$} & \multicolumn{1}{c}{$\alpha$} \\
\hline Exponential & 1 & 23,040 & 169,175 & 163,384 & 0.56 \\
& 1 & 18,720 & 305,119 & 27,440 & 0.09 \\
Lognormal & 0.8 & 23,040 & 97,543 & 235,016 & 0.81 \\
& 1 & 23,040 & 118,124 & 214,435 & 0.74 \\
& 1.2 & 23,040 & 141,205 & 191,354 & 0.66 \\
& 0.8 & 18,720 & 125,453 & 207,106 & 0.72 \\
& 1 & 18,720 & 159,500 & 173,059 & 0.60 \\
& 1.2 & 18,720 & 198,963 & 133,596 & 0.46 \\
\hline
\end{tabular}

The demand model's parameters are derived from the California Energy Commission's report on state energy demand forecasts between the years 2012 and 2022 [6] and statistics obtained from the California Center for Sustainable Energy (CCSE)'s website. ${ }^{9}$ The California Energy Commission's report tabulates the projected number of BEVs and PHEVs on the road for low scenario and high scenario cases for selected years between 2011 and 2022. Accordingly, the forecasts of the relevant periods are listed in Table 1.

The CCSE's website offers interactive access to data and analyses of the CVRP project. From the database accessed at the end of January 2014, we determine that the rebates issued under this program as of the end of 2013 were approximately $\$ 95$ million for 45,000 vehicles (note that we only consider rebates given to ZEVs and PHEVs in this calculation). Thus, the realized rebate is $\$ 2116$ per vehicle for the current case in addition to the federal tax credit of $\$ 7500$.

We utilize the current structure of the intervention mechanism and realized outcomes to set the demand model's parameters $\left(\mu_{\infty}\right.$ and $d$ in Eq. (14)) for the three years period of 2011 through 2013 , and set the salvage value to its final values. As noted earlier, $r$ is $\$ 2116$ per vehicle and sales up to end of 2013 were 45,000 . By setting expected sales equal to 45,000 we obtain a condition that yields a relationship between $s$ and $\mu_{\infty}-d$ when solved together with the newsvendor optimality condition, particularly for each distribution. Note that these conditions are determined under the assumption that no investment is made in battery development during these years (i.e. $B_{d}=0$ ). Note that this is consistent with what is reported on the pp 6-7 of [36]. However, we can also interpret the value of $B_{d}$ as additional investment, if the current policy includes investment for battery development. We test our model for both low and high salvage values, which are $65 \%$ and $80 \%$ of the MSRP value, respectively. Following the conditions we derived, $s$ and $\mu_{\infty}-d$ pairs considered for the current structure appear to be as tabulated in Table 2. Next, we find $d$ values corresponding to these pairs by fixing $\mu_{\infty}$ to the total forecast of the high scenario for these years, 332,559. Notice that as the salvage value increases so does $d$. This result indicates that the potential to improve demand by investing in battery development is high for larger salvage values. Moreover, we assume the values of $a$ and $k$ to be, respectively, 1.1 and $10^{-8}$ throughout the analysis.

\footnotetext{
${ }^{9}$ http://energycenter.org/clean-vehicle-rebate-project/cvrp-project-statistics.
} 
Suppose that the low scenario occurs with probability $\alpha$. Combining the estimate of $\mu_{\infty}-d$, and the yearly forecasts in years 2011 and 2013 for the low and high scenarios (i.e. $\left.\alpha(43,113)+(1-\alpha)(332,559)=\mu_{\infty}-d\right)$, we obtain $\alpha$ values, as in Table 2. Assuming that the same state of the world is preserved, we use $\alpha$ values to estimate the demand parameters of future periods for each particular data set. Similar to Table 2 above, we present the demand model's parameters to be used for mediumand long-term scenarios in Appendix B.

To determine the monetary value per expected sales $(\beta)$, we use a version of the proposed model to calibrate the parameter. We specifically solve a subproblem of Model JM, which is provided in Appendix $C$. This subproblem decides on $B_{d}, B_{r}, r$, and $Q$ with the objective of maximizing expected sales for a given budget level. Note that the constraints are similar to those in Model JM. As input, we rely on the cost and demand model's data described above and we consider the budget spent as of the end of 2013 (\$95 million). We solve the subproblem for exponential distribution and lognormal distribution with varying coefficient of variation values. Next, we extract the Lagrange multiplier value of constraint (C.2), which gives us the marginal increase in the expected sales from a unit relaxation of the budget. Note that reciprocal of the Lagrange multiplier corresponds to the monetary value of expected sales, $\beta$. We report the estimated $\beta$ values for each case in Table $\mathrm{C} 1$ in Appendix C. To be inclusive, we consider the range for $\beta$ as [32005100], and use the upper and lower limit, and intermediate value of the range in the numerical studies, which are $\{3200$, 4150, 5100\}.

We conduct three sets of analyses for exponential distribution and lognormal distribution with varying coefficient of variation values: (1) base-case scenario (current structure) using the estimates discussed in this subsection, (2) medium-term scenario including the years 2011 through 2015, and (3) long term scenario including the years 2011 through 2022.

Before continuing with the numerical results, we list the parameters that take the same values for each scenario: $p=26,300$, $c=26,986, a=1.1$, and $k=10^{-8}$. Note that $p=26,300$ considers 18,800 , amount customer is willing to pay, plus 7500 tax credit that comes from the federal government, which is not a part of California's budget. Further, if lognormal distribution is used to represent the demand, we restrict our analysis for constant coefficient of variation.

\subsection{Main results}

The cases considered in the computational study are labeled and summarized in Table 3. Notice that the letters in labels are assigned in the order of increasing values of $\beta$ first, then in increasing values of coefficient of variation. For each set, we tabulate the results in Appendix D for the joint mechanism (JM), and also for the decentralized (DA) and base decentralized (BDA) approaches, which can be regarded as benchmarks. The tables include utility values, expected sales, details of the mechanisms, mean demand, and expected profit of the retailer, which is calculated by expression (7). For the case study, we solve the decentralized and base decentralized approaches with a given rebate of $\$ 2116$, which is the current realized rebate in California. A no-intervention case cannot be employed as a benchmark for this case, because $c>p$ and rebate must be given for the system to operate. It is necessary to reemphasize that the quantities presented throughout the study should not be interpreted as the number of vehicles to be manufactured, but as the capacity plan of the retailer.

\subsubsection{Base-case scenario (2011-2013)}

For this scenario set, we consider cases $1 \mathrm{a}$ through $4 \mathrm{i}$ and demonstrate their associated results in Tables D1-D3 in Appendix D.

When exponential distribution is used to represent demand, we observe that the joint mechanism is not applied in general, mainly due to the structure of distribution and the cost values. Since $c>p$ for the vehicles and uncertainty is very high, the central authority gives priority to increasing the vehicle's profitability to encourage the retailer to order more. However, when lognormal distribution is under consideration, the joint mechanism is implemented for most of the cases.

For this scenario set, we report the percentage improvement obtained by implementing the joint mechanism compared to the current policy (Model BDA) for the medium $\beta$ value in Table 4 . The percentage values indicate that the percentage utility loss is minimal, less than $1 \%$, when demand distribution is exponential. Thus, we can say that the current policy is reasonable if exponential distribution is a better candidate to represent the demand. On the other hand, the joint mechanism performs better than the current policy under lognormal distribution.

One can also see from the solutions that the changes in results are as expected in response to the changes in $\beta$. Particularly, utility, expected sales, rebate amount, quantity ordered, and the retailer's

Table 4

Percentage improvement of the utility with respect to current policy when $\beta=4150$.

\begin{tabular}{|c|c|c|c|c|}
\hline \multirow[t]{2}{*}{$\mathrm{cV}$} & \multicolumn{2}{|l|}{$s=\$ 23,040$} & \multicolumn{2}{|l|}{$s=\$ 18,720$} \\
\hline & Exp. dist. (\%) & Logn. dist. (\%) & Exp. dist. (\%) & Logn. dist. (\%) \\
\hline 0.8 & & 47.0 & & 19.8 \\
\hline 1 & 0.03 & 20.1 & 0.8 & 5.4 \\
\hline 1.2 & & 6.5 & & 3.0 \\
\hline
\end{tabular}

Table 3

Cases considered in the computational study.

\begin{tabular}{|c|c|c|c|c|c|}
\hline Case no. & Distribution & cv & Horizon & $\mathbf{s}(\$)$ & $\beta(\$)$ \\
\hline $1 a, 1 b, 1 c$ & Exponential & 1 & Base case & 23,040 & $\{3200,4150,5100\}$ \\
\hline $2 a, 2 b, 2 c$ & Exponential & 1 & Base case & 18,720 & $\{3200,4150,5100\}$ \\
\hline $3 a-3 i$ & Lognormal & $\{0.8,1,1.2\}$ & Base case & 23,040 & $\{3200,4150,5100\}$ \\
\hline $4 a-4 i$ & Lognormal & $\{0.8,1,1.2\}$ & Base case & 18,720 & $\{3200,4150,5100\}$ \\
\hline $5 a, 5 b, 5 c$ & Exponential & 1 & Medium term & 23,040 & $\{3200,4150,5100\}$ \\
\hline $6 a, 6 b, 6 c$ & Exponential & 1 & Medium term & 18,720 & $\{3200,4150,5100\}$ \\
\hline $7 a-7 i$ & Lognormal & $\{0.8,1,1.2\}$ & Medium term & 23,040 & $\{3200,4150,5100\}$ \\
\hline $8 a-8 i$ & Lognormal & $\{0.8,1,1.2\}$ & Medium term & 18,720 & $\{3200,4150,5100\}$ \\
\hline $9 a, 9 b, 9 c$ & Exponential & 1 & Long term & 23,040 & $\{3200,4150,5100\}$ \\
\hline $10 a, 10 b, 10 c$ & Exponential & 1 & Long term & 18,720 & $\{3200,4150,5100\}$ \\
\hline $11 a-11 i$ & Lognormal & $\{0.8,1,1.2\}$ & Long term & 23,040 & $\{3200,4150,5100\}$ \\
\hline $12 a-12 i$ & Lognormal & $\{0.8,1,1.2\}$ & Long term & 18,720 & $\{3200,4150,5100\}$ \\
\hline
\end{tabular}


expected profit are increasing in increasing $\beta$ values. Also, for the cases that implement the joint mechanism, $B_{d}$ increases if $\beta$ increases, which consequently makes the joint mechanism more valuable. In summary, as very much expected, the adoption of vehicles is accelerated with an increase in the benefits to society per unit of expected sales.

Note that we can explore the problem by analyzing Pareto optimal curve along which optimal budget and expected sales or utility are shown. Fig. 1a and b in Appendix E depict Pareto optimal curves for one of the parameter settings.

\subsubsection{Medium- (2011-2015) and long-term scenarios (2011-2022)}

The details of the solutions for the cases considered can be found in Tables D4-D6 and in Tables D7-D9 in Appendix D for medium- and long-term, respectively.

After analyzing all scenarios, we see that the value of the joint mechanism in terms of utility is substantial for the cases with high $s$ or $d$ values. Moreover, we observe that the value of the joint mechanism is especially recognized in long-term planning (see Table 5). Long-term planning allows one to clearly see the evolution of demand by the investment amount and thus helps the central authority better manage the budget.

The other observations and findings related to mechanism performance are similar to the base-case.

\subsubsection{Consistency check with US administration targets}

We perform the medium-term scenario analysis to observe whether the 2015 goals ${ }^{10}$ are met under the mild assumption that the same state of the world is preserved. As discussed earlier, California constitutes $10 \%$ of the total car market in 2013. Thus, using $10 \%$ the goal for our case emerges as having about 100,000 EVs on the road in California by 2015. On the other hand, 2013 statistics on EV purchases reveal that $40 \%$ of sales are in California. The results obtained by joint mechanism take values between 100,000 and 400,000 , which imply that the numbers obtained by the proposed mechanism are within the $10-40 \%$ bound. This can be interpreted as follows: as the number of vehicles on the road increases, one would expect California dominance on sales percentage to drop, as there will be increasing sales in other US states as well.

Moreover, according to an article published in New York Times, ${ }^{11}$ there are 150,000 electric vehicles in California by the end of 2015. Expected sales values found by base decentralized approach (reflecting the current practice) in the medium term are very close to the realized sales, i.e. in the range of $[134,600,146,600]$, which can be considered as another benchmark validating our parameter estimations.

\subsection{Analysis of results}

In this section, we use the numerical results provided in the previous section as a basis to further investigate two main issues: (1) the impact of uncertainty on the solutions, and (2) expected excess budget required.

\subsubsection{Impact of uncertainty}

The results of the lognormal distribution case indicate that demand uncertainty can have a huge impact on the results, hence this leads us to investigate the impact of uncertainty on four specific outcomes: (i) rebate amounts issued, (ii) the retailer's expected profit, (iii) utility attained, and (iv) percentage

\footnotetext{
10 http://www.whitehouse.gov/the-press-office/2011/01/25/remarks-pre sident-state-union-address.

11 http://www.nytimes.com/2015/12/01/science/electric-car-auto-dealers. html?smid = nytcore-ipad-share\&smprod $=$ nytcore-ipad\&_r $=0$.
}

improvement compared to base decentralized approach. We report the analysis for only one salvage value, i.e. $\$ 23,040$, at the medium $\beta$ level, i.e. 4150 , since the findings are similar to other combinations of salvage value and $\beta$ values. Tables $6-10$ show how each of these outcomes varies with the level of uncertainty under different horizons.

- Rebate amount: The impact of the increase in demand variability is in two levels, i.e. it decreases the potential to improve demand ( $d$ value) while increasing the retailer's risks of overordering and under-ordering. These conditions thus result in

Table 5

Percentage improvement of the utility with respect to current policy in the longterm when $\beta=4150$.

\begin{tabular}{|c|c|c|c|c|}
\hline \multirow[t]{2}{*}{$\mathrm{cV}$} & \multicolumn{2}{|l|}{$s=\$ 23,040$} & \multicolumn{2}{|l|}{$s=\$ 18,720$} \\
\hline & Exp. dist. (\%) & Logn. dist. (\%) & Exp. dist. (\%) & Logn. dist. (\%) \\
\hline 0.8 & & 123.7 & & 85.0 \\
\hline 1 & 34.7 & 87.5 & 0.8 & 49.2 \\
\hline 1.2 & & 60.2 & & 24.8 \\
\hline
\end{tabular}

Table 6

Rebate amounts (\$) when $\beta=4150$ and $s=23,040$.

\begin{tabular}{|c|c|c|c|c|c|c|}
\hline \multirow[t]{2}{*}{$\mathrm{cv}$} & \multicolumn{2}{|l|}{ Base case } & \multicolumn{2}{|c|}{ Medium term } & \multicolumn{2}{|l|}{ Long term } \\
\hline & Exp. dist. & Logn. dist. & Exp. dist. & Logn. dist. & Exp. dist. & Logn. dist. \\
\hline 0.8 & & 1569 & & 1569 & & 1569 \\
\hline 1 & 2147 & 1689 & 2147 & 1689 & 2147 & 1689 \\
\hline 1.2 & & 1785 & & 1785 & & 1785 \\
\hline
\end{tabular}

Table 7

Retailer's expected profit $\left(\$\left(\times 10^{6}\right)\right)$ when $\beta=4150$ and $s=23,040$.

\begin{tabular}{|c|c|c|c|c|c|c|}
\hline \multirow[t]{2}{*}{$\mathrm{cV}$} & \multicolumn{2}{|l|}{ Base case } & \multicolumn{2}{|c|}{ Medium term } & \multicolumn{2}{|l|}{ Long term } \\
\hline & Exp. dist. & Logn. dist. & Exp. dist. & Logn. dist. & Exp. dist. & Logn. dist. \\
\hline 0.8 & & 49.6 & & 215.7 & & 822.7 \\
\hline 1 & 36.9 & 42.7 & 171.8 & 192.3 & 674.7 & 743.0 \\
\hline 1.2 & & 36.8 & & 170.1 & & 663.8 \\
\hline
\end{tabular}

Table 8

Utility $\left(\$\left(\times 10^{6}\right)\right)$ when $\beta=4150$ and $s=23,040$.

\begin{tabular}{|c|c|c|c|c|c|c|}
\hline \multirow[t]{2}{*}{$\mathrm{cv}$} & \multicolumn{2}{|l|}{ Base case } & \multicolumn{2}{|c|}{ Medium term } & \multicolumn{2}{|l|}{ Long term } \\
\hline & Exp. dist. & Logn. dist. & Exp. dist. & Logn. dist. & Exp. dist. & Logn. dist. \\
\hline 0.8 & & 134.5 & & 656.2 & & 2833.9 \\
\hline 1 & 91.6 & 109.9 & 351.1 & 515.1 & 1487.3 & 2244.2 \\
\hline 1.2 & & 97.5 & & 422.1 & & 1830.9 \\
\hline
\end{tabular}

Table 9

Percentage improvement of utility achieved by joint mechanism with respect to base decentralized approach (\%) when $\beta=4150$ and $s=23,040$.

\begin{tabular}{|c|c|c|c|c|c|c|}
\hline \multirow[t]{2}{*}{$\mathrm{cV}$} & \multicolumn{2}{|c|}{ Base case (\%) } & \multicolumn{2}{|c|}{ Medium term (\%) } & \multicolumn{2}{|c|}{ Long term (\%) } \\
\hline & Exp. dist. & Logn. dist. & Exp. dist. & Logn. dist. & Exp. dist. & Logn. dist. \\
\hline 0.8 & & 47.0 & & 139.6 & & 123.7 \\
\hline 1 & 0.03 & 20.1 & 20.9 & 84.1 & 34.7 & 87.5 \\
\hline 1.2 & & 6.5 & & 48.5 & & 60.2 \\
\hline
\end{tabular}


larger rebate amounts for higher coefficient of variation values. Moreover, we observe that the rebate amounts remain constant with respect to changes in the horizon length as shown in Corollary 3.1.

- Retailer's expected profit: The values given in Table 7 are the retailer's expected profit for the whole horizon of the associated scenario. When comparing the expected profit for varying demand uncertainty levels, as expected profit is smaller for higher values of the coefficient of variation. This observation implies that the retailer suffers from increasing levels of demand uncertainty in terms of expected profit.

- Utility and percentage improvement with respect to the current policy: The best results in terms of utility (or utility per year, which can roughly be found by dividing the numbers in Table 8 by the number of years considered in the corresponding case) appears when the coefficient of variation is lowest. Moreover, the results in Table 9 display the negative impact of uncertainty on the performance of the joint mechanism relative to the current policy (base decentralized approach). The reason behind this finding is that the investment made in battery development only affects the mean of the demand while increasing the variation proportionally. However, if a demand-increasing strategy that will also reduce variability is implemented, the joint mechanism will be more effective for higher demand values.

Another benchmark to use would be decentralized approach (Model DA). Model DA allows for spending on demand-increasing strategies, while setting the rebate value to its current level. Percentage improvement of utility achieved by Model $D A$ relative to current practice is presented in Table 10. Note that considering both intervention strategies, even one of them is set with respect to other considerations, will improve today's solution.

\subsubsection{Expected excess budget required}

We express the budget constraint on the amount of rebates (Eq. (9)) in terms of expected sales. This formulation may lead to the discovery that the actual cost of rebates is higher than $B_{r}$ once the demand is realized. Thus, we quantify the expected excess budget required to check whether the amounts are reasonable.

\section{Table 10}

Percentage improvement of utility achieved by decentralized approach with respect to base decentralized approach (\%) when $\beta=4150$ and $s=23,040$.

\begin{tabular}{|c|c|c|c|c|c|c|}
\hline \multirow[t]{2}{*}{$\mathrm{cV}$} & \multicolumn{2}{|c|}{ Base case (\%) } & \multicolumn{2}{|c|}{ Medium term (\%) } & \multicolumn{2}{|c|}{ Long term (\%) } \\
\hline & Exp. dist. & Logn. dist. & Exp. dist. & Logn. dist. & Exp. dist. & Logn. dist. \\
\hline 0.8 & & 32.1 & & 117.8 & & 105.7 \\
\hline 1 & 0.0 & 12.8 & 20.8 & 73.4 & 34.7 & 77.8 \\
\hline 1.2 & & 2.9 & & 43.1 & & 55.0 \\
\hline
\end{tabular}

Defining $\mathbb{E}[E B]$ as expected excess budget required, we measure it by

$\mathbb{E}[E B]=r \times\left[\int_{E[\text { sales }]}^{Q}(x-E[\right.$ sales $]) f_{B_{d}}(x) d x+\int_{Q}^{\infty}(Q-E[$ sales $\left.]) f_{B_{d}}(x) d x\right]$.

Note that the expected budget overflow is equal to this amount.

Table 11 displays the expected excess budget required and its percentage relative to the applicable budget in parentheses, respectively. Again, we only show the results for the salvage value of $\$ 23,040$ at the medium $\beta$ value for the three mechanisms considered. Recall that the rebates issued under the joint mechanism are less in relation to the decentralized and base decentralized approaches, which is why in general the percentage of $\mathbb{E}[E B]$ relative to budget is larger for the decentralized and base decentralized approaches. The results indicate that the government has more risk if it regulates the system by a single intervention tool. Moreover, if we evaluate the expected excess budget required on the basis of the percentage of budget under consideration, we observe that the percentages vary from $3.1 \%$ to $12.4 \%$. Thus, we can conclude that the expected excess budget required does not appear to be too large relative to the government's budget.

\section{Conclusion}

In this paper, we study the problem of designing an intervention mechanism for public-interest goods. More specifically, we consider a system composed of a retailer and a central authority that regulates the system through a joint mechanism in which demand-increasing strategies and rebates are used. The main goal of the intervention is to encourage the retailer to make decisions that would best benefit the system. By formulating the system via bilevel programming, we do not solely maximize utility, but also consider the retailer's expected profit. We characterize the structure of the solution and show that the rebate amount may be independent of the investment made in demand-increasing strategies and improvement pattern of mean demand.

In this study, we define expected excess budget that plays a supportive role in decisions to be made. We believe that with stochastic constraints, such quantities should be computed to reflect possible risks in the decisions made.

We attempt to validate the benefits of our model with available data from the California electric vehicle market. The results of this case study demonstrate that the joint mechanism brings considerable benefits in terms of utility.

Another important finding of the case study is that when the problem is solved considering longer horizons, the proposed joint mechanism exploits the range of possibilities more extensively.

Table 11

Expected excess budget required $\left(\$\left(\times 10^{6}\right), \%\right)$ when $\beta=4150$ and $s=23,040$.

\begin{tabular}{|c|c|c|c|c|c|c|c|}
\hline \multirow[t]{2}{*}{ Mechanism } & \multirow[t]{2}{*}{$\mathrm{cV}$} & \multicolumn{2}{|l|}{ Base case } & \multicolumn{2}{|c|}{ Medium term } & \multicolumn{2}{|l|}{ Long term } \\
\hline & & Exp. dist. & Logn. dist. & Exp. dist. & Logn. dist. & Exp. dist. & Logn. dist. \\
\hline JM & $\begin{array}{l}0.8 \\
1 \\
1.2\end{array}$ & $(12.1,12.4 \%)$ & $\begin{array}{c}(5.3,3.1 \%) \\
(5.8,4.3 \%) \\
(6.0,5.7 \%)\end{array}$ & $(56.6,10.6 \%)$ & $\begin{array}{l}(23.3,3.4 \%) \\
(26.2,4.4 \%) \\
(27.6,5.4 \%)\end{array}$ & $(222.1,11.2 \%)$ & $\begin{array}{l}(88.7,3.9 \%) \\
(101.2,5.0 \%) \\
(107.6,6.0 \%)\end{array}$ \\
\hline Dec. & $\begin{array}{l}0.8 \\
1 \\
1.2\end{array}$ & $(11.6,12.2 \%)$ & $\begin{array}{l}(12.3,5.3 \%) \\
(11.0,6.3 \%) \\
(9.8,7.5 \%)\end{array}$ & $(54.1,10.4 \%)$ & $\begin{array}{l}(54.5,5.6 \%) \\
(50.3,6.4 \%) \\
(45.5,7.1 \%)\end{array}$ & $(212.3,11.0 \%)$ & $\begin{array}{c}(209.5,6.1 \%) \\
(195.2,7.0 \%) \\
(178.0,7.6 \%)\end{array}$ \\
\hline Base Dec. & $\begin{array}{l}0.8 \\
1 \\
1.2\end{array}$ & $(11.6,12.2 \%)$ & $\begin{array}{c}(6.5,6.8 \%) \\
(7.4,7.8 \%) \\
(8.1,8.5 \%)\end{array}$ & $(36.9,12.2 \%)$ & $\begin{array}{l}(19.4,6.8 \%) \\
(22.6,7.8 \%) \\
(25.2,8.5 \%)\end{array}$ & $(140.0,12.2 \%)$ & $\begin{array}{r}(89.9,6.8 \%) \\
(96.7,7.8 \%) \\
(101.3,8.5 \%)\end{array}$ \\
\hline
\end{tabular}


Also, demand variability turns out to be an extremely important factor for the environment considered in the case study. Based on our results, we conclude that approaches that do not consider demand variability may lead to erroneous decisions. This finding is especially apparent in the decision on rebate quantity, as well as in the expected budget that would be required by the central authority.

Note that this modeling framework can be modified for alternative intervention mechanisms, i.e. a subsidy given to the retailer per unit ordered or direct investment affecting any other parameter of the system. Since both mechanisms achieve similar results, we do not analyze them in depth. This possibility allows the central authority the ability to formulate a bilevel program for each intervention scheme and compare the effect of each scheme. Another improvement can be to represent more than one factor that will change the mean demand, each factor requiring a separate budget. A good example can be depicted from [26]: R\&D efficiency is not only a function of research expenditure, but also research manpower. Hence, more realistic representations can be used to replace Eqs. (14) and (20).

\section{Appendix A. Proofs}

Proof of Corollary 3.1. Considering (16), $Q=F_{B_{d}}^{-1}\left(\frac{p+r-c}{p+r-s}\right)$ and $1-F_{B_{d}}(Q)=\left(\frac{c-s}{p+r-s}\right)$. Hence, we can express expected sales as:

$\mathbb{E}\left[\min \{Q, D\} \mid B_{d}\right]=\int_{0}^{F_{B_{d}}^{-1}\left(\frac{p+r-c}{p+r-s}\right)} x f_{B_{d}}(x) d x+\left(\frac{c-s}{p+r-s}\right) F_{B_{d}}^{-1}\left(\frac{p+r-c}{p+r-s}\right)$

Using (A.1) for the expected sales, the objective function can be written as:

$u\left(B_{d}, r\right)=(\beta-r)\left\{\int_{0}^{F_{B_{d}}^{-1}\left(\frac{p+r-c}{p+r-s}\right)} x f_{B_{d}}(x) d x+\left(\frac{c-s}{p+r-s}\right) F_{B_{d}}^{-1}\left(\frac{p+r-c}{p+r-s}\right)\right\}-B_{d}$

Next, we consider first order condition of (A.2) with respect to $r$, which is given below:

$$
\begin{gathered}
\int_{0}^{F_{B_{d}}^{-1}\left(\frac{p+r-c}{p+r-s}\right)} \chi f_{B_{d}}(x) d x+\left(\frac{c-s}{p+r-s}\right) F_{B_{d}}^{-1}\left(\frac{p+r-c}{p+r-s}\right) \\
\quad=(\beta-r) \frac{1}{f_{B_{d}}\left(F_{B_{d}}^{-1}\left(\frac{p+r-c}{p+r-s}\right)\right)} \frac{(c-s)^{2}}{(p+r-s)^{3}}
\end{gathered}
$$

After evaluating (A.3) for exponential and lognormal distribution and rearranging, it can be shown that optimal rebate is independent of $\mu\left(B_{d}\right)$ for the mentioned distributions.

(i) Assume that demand is exponentially distributed with mean $\mu\left(B_{d}\right)$. Then, (A.3) can be written as:

$$
\begin{aligned}
& \mu\left(B_{d}\right)\left(\frac{c-s}{p+r-s}\right) \ln \left(\frac{c-s}{p+r-s}\right)+\mu\left(B_{d}\right)\left(\frac{p+r-c}{p+r-s}\right) \\
& \quad-\mu\left(B_{d}\right)\left(\frac{c-s}{p+r-s}\right) \ln \left(\frac{c-s}{p+r-s}\right)=(\beta-r) \mu\left(B_{d}\right) \frac{c-s}{(p+r-s)^{2}}
\end{aligned}
$$

After rearranging (A.4), one can show that $r$ can be found by $\sqrt{\beta(c-s)+c(p-s)-s(p-s)}-(p-s)$, which is independent of $B_{d}$ and $\mu\left(B_{d}\right)$.

(ii) Assume that demand is lognormally distributed with parameters $(v, \tau)$ and mean $\mu\left(B_{d}\right)$. Note that $\mu\left(B_{d}\right)=e^{v+\tau^{2} / 2}$. Given the optimal solution of the newsvendor problem, $Q=$ $F_{B_{d}}^{-1}\left(\frac{p+r-c}{p+r-s}\right)=e^{v+\tau z}$, where $z=\Phi^{-1}\left(\frac{p+r-c}{p+r-s}\right)$. Then, (A.3) can be expressed as follows:

$$
\begin{aligned}
e^{v+\tau^{2} / 2} \Phi(z-\tau)+e^{v+\tau z}\left(\frac{c-s}{p+r-s}\right) \\
=(\beta-r) e^{v+\tau z} \tau \sqrt{2 \pi} e^{-z^{2} / 2} \frac{(c-s)^{2}}{(p+r-s)^{3}}
\end{aligned}
$$

Using $\mu\left(B_{d}\right)=e^{v+\tau^{2} / 2}$, one can show that (A.5) can be expressed independent of $\mu\left(B_{d}\right)$ and the result follows. $\square$

Proof of Corollary 3.2. This proof is similar to that of Corollary 3.1. Specifically, evaluating (A.3) with mean demand function given in (14) and coefficient of variation function given in (20), and rearranging give the result. Of course, in this case $B_{d}$ cannot be eliminated in (A.3).

\section{Appendix B. Demand model's parameters for medium- and long-term scenarios}

See Tables B1 and B2.

Table B2

Demand model's parameters $\left(\mu_{\infty}, d\right)$ for long-term scenario.

\begin{tabular}{|c|c|c|c|c|}
\hline \multirow[t]{2}{*}{$\mathrm{cv}$} & \multicolumn{2}{|l|}{$s=\$ 23,040$} & \multicolumn{2}{|l|}{$S=\$ 18,720$} \\
\hline & Exp. dist. & Logn. dist. & Exp. dist. & Logn. dist. \\
\hline 0.8 & & $(1,081,703,789,863)$ & & $(1,081,703,696,060)$ \\
\hline 1 & $(1,081,703,544,766)$ & $(1,081,703,720,692)$ & $(1,081,703,87,552)$ & $(1,081,703,581,632)$ \\
\hline 1.2 & & $(1,081,703,643,120)$ & & $(1,081,703,449,001)$ \\
\hline
\end{tabular}

\begin{tabular}{cccccc}
\hline \multirow{2}{*}{ cv } & $s=\$ 23,040$ & & & $s=\$ 18,720$ & \\
\cline { 2 - 3 } \cline { 5 - 5 } \cline { 5 - 5 } & Exp. dist. & Logn. dist. & & Exp. dist. & Logn. dist. \\
\hline \multirow{2}{*}{0.8} & & $(3,574,670$, & & $(3,574,670$, \\
& & $2,224,893)$ & & $1,960,669)$ \\
1 & $(3,574,670$, & $(3,574,670$, & & $(3,574,670$, & $(3,574,670$, \\
& $1,534,501)$ & $2,030,053)$ & & $246,616)$ & $1,638,347)$ \\
1.2 & & $(3,574,670$, & & $(3,574,670$, \\
& & $1,811,545)$ & & $1,264,751)$ \\
\hline
\end{tabular}

The numbers are presented with precision so that they can be used for performing explicit calculations for comparison if needed.

Table B1

Demand model's parameters $\left(\mu_{\infty}, d\right)$ for medium-term scenario. 
Appendix C. Subproblem used to calibrate possible values for $\beta$

\section{Subproblem:}

$\max _{r, B_{d}, B_{r}, Q} \mathbb{E}\left[\min \{Q, D\} \mid B_{d}\right]$

s.t. $\quad B_{d}+B_{r} \leq B$

$r \mathbb{E}\left[\min \{Q, D\} \mid B_{d}\right] \leq B_{r}$

$r \geq c-p$

$r \geq 0$

$B_{d}, B_{r} \geq 0$

$F_{B_{d}}(Q)=\frac{p+r-c}{p+r-s}$

Proposition C.1. Consider the objective function given in (C.1). Then, the following relationships hold at the optimal solution, $\left(r^{*}, Q^{*}, B_{d}^{*}, B_{r}^{*}\right)$ :

a) $r^{*} \mathbb{E}\left[\min \left\{Q^{*}, D\right\} \mid B_{d}\right]=B_{r}^{*}$,

b) $B_{d}^{*}+B_{r}^{*}=B$.

Proof. (a) Suppose to the contrary that there exists some solution $\left(\bar{r}, \bar{Q}, B_{d}^{*}, B_{r}^{*}\right)$ such that $\bar{r} \neq r^{*}, \bar{Q} \neq Q^{*}, \bar{r} E\left[\min \{\bar{Q}, D\} \mid B_{d}=B_{d}^{*}\right]<B_{r}^{*}$, and it has a higher objective function value. Assume that we keep $B_{d}^{*}$ and $B_{r}^{*}$ constant and increase $\bar{r}$ by $\epsilon$, then we will arrive at a

Table C1

Calibrated $\beta$ values.

\begin{tabular}{|c|c|c|c|c|}
\hline \multirow[t]{2}{*}{$\mathrm{cV}$} & \multicolumn{2}{|c|}{$s=\$ 23,040$} & \multicolumn{2}{|c|}{$s=\$ 18,720$} \\
\hline & Exp. dist. & Logn. dist. & Exp. dist. & Logn. dist. \\
\hline 0.8 & & 3132 & & 3802 \\
\hline 1 & 4058 & 3582 & 3788 & 4487 \\
\hline 1.2 & & 4012 & & 5161 \\
\hline
\end{tabular}

solution $\left(\widehat{r}, \widehat{Q}, B_{d}^{*}, B_{r}^{*}\right)$, for which $\widehat{Q}>\bar{Q}$ by the monotonicity of $F_{B_{d}}$. Since the objective function is increasing concave with respect to $Q$ the new solution will give a higher objective value, which contradicts with optimality. Continuing in this manner, i.e. increasing $r$ by small increments, we will reach $\left(r^{*}, Q^{*}, B_{d}^{*}, B_{r}^{*}\right)$. The result then follows.

b) We follow the same logic as in part (a). Suppose for a contradiction that there exists some solution $\left(\bar{r}, \bar{Q}, \overline{B_{d}}, \overline{B_{r}}\right)$ such that $\overline{B_{d}}+\overline{B_{r}}<B$, and it has a higher objective function value. This implies that either $\overline{B_{d}}<B_{d}^{*}$ or $\overline{B_{r}}<B_{r}^{*}$, or both.

When $\overline{B_{d}}<B_{d}^{*}$; if we increase $\overline{B_{d}}$ by $\epsilon$, we will arrive at a solution $\left(\bar{r}, \widehat{Q}, \widehat{B_{d}}, \overline{B_{r}}\right)$ for which $\widehat{Q}>\bar{Q}$ by the first order stochastic dominance order of cdfs. Since the objective function is increasing with respect to $Q$ and $B_{d}$, the new solution will result in a higher objective function value, which contradicts with optimality.

When $\overline{B_{r}}<B_{r}^{*}$; if we increase $\overline{B_{r}}$ by $\epsilon, \bar{r}$ and $\bar{Q}$ also increase by part (a). Now, we arrive at a new solution $\left(\tilde{r}, \tilde{Q}, \overline{B_{d}}, \tilde{B_{r}}\right)$, for which $\tilde{r}>\bar{r}$ and thus $\tilde{Q}>\bar{Q}$ by the monotonicity of cdf. $\tilde{Q}$ has a higher objective function value, which contradicts with optimality.

Last, if both $\overline{B_{d}}<B_{d}^{*}$ and $\overline{B_{r}}<B_{r}^{*}$, we can arrive at a better solution by playing with $B_{d}$ and $B_{r}$ values in the same manner as in the previous cases, and this contradicts with the optimality of $\left(\bar{r}, \bar{Q}, \overline{B_{d}}, \overline{B_{r}}\right)$. In each of the three cases, by continuing to increase the values of $B_{d}$ or/and $B_{r}$, we can achieve the optimal solution $\left(r^{*}, Q^{*}, B_{d}^{*}, B_{r}^{*}\right)$. The result then follows.

Note that one can solve the subproblem using the tightness of the budget-related constraints, (C.2) and (C.3).

\section{Appendix D. Computational results}

We solve our models by the nonlinear solver CONOPT within the GAMS environment. We also perform runs with different nonlinear solvers included in GAMS environment, such as MINOS, BARON, KNITRO, etc. However, we attempt to solve our data instances with CONOPT because it produces more reliable results. The solutions are obtained very quickly, in approximately $0.004 \mathrm{~s}$, because the problems are not very large.

Table D1

Solutions of base-case scenario for cases 1 and 2 .

\begin{tabular}{|c|c|c|c|c|c|c|c|c|c|c|}
\hline $\begin{array}{l}\text { Case } \\
\mathrm{Nb} .\end{array}$ & $\begin{array}{l}\beta \\
(\$)\end{array}$ & Mechanism & $\begin{array}{l}\text { Objective } \\
\left(\$ \times 10^{6}\right)\end{array}$ & $\begin{array}{l}\text { Exp. sales } \\
\left(\times 10^{3} \text { vehicles }\right)\end{array}$ & $\begin{array}{l}Q \\
\left(\times 10^{3} \text { vehicles }\right)\end{array}$ & $\begin{array}{l}r \\
(\$)\end{array}$ & $\begin{array}{l}B_{d} \\
\left(\$ \times 10^{6}\right)\end{array}$ & $\begin{array}{l}B_{r} \\
\left(\$ \times 10^{6}\right)\end{array}$ & $\begin{array}{l}E[P(Q)] \\
\left(\$ \times 10^{6}\right)\end{array}$ & $\begin{array}{l}\mu\left(B_{d}\right) \\
\left(\times 10^{3} \text { vehicles }\right)\end{array}$ \\
\hline \multirow[t]{3}{*}{ 1a } & \multirow[t]{3}{*}{3200} & JM & 52.1 & 37.0 & 41.7 & 1789 & 0.0 & 66.1 & 22.0 & 169.2 \\
\hline & & DA & 48.8 & 45.0 & 52.3 & 2116 & 0.0 & 95.2 & 35.5 & 169.2 \\
\hline & & BDA & 48.8 & 45.0 & 52.3 & 2116 & 0.0 & 95.2 & 35.5 & 169.2 \\
\hline \multirow[t]{3}{*}{$1 \mathrm{~b}$} & \multirow[t]{3}{*}{4150} & JM & 91.6 & 45.7 & 53.3 & 2147 & 0.0 & 98.2 & 36.9 & 169.2 \\
\hline & & DA & 91.5 & 45.0 & 52.3 & 2116 & 0.0 & 95.2 & 35.5 & 169.2 \\
\hline & & BDA & 91.5 & 45.0 & 52.3 & 2116 & 0.0 & 95.2 & 35.5 & 169.2 \\
\hline \multirow[t]{3}{*}{$1 \mathrm{c}$} & \multirow[t]{3}{*}{5100} & JM & 142.8 & 62.3 & 74.7 & 2484 & 20.2 & 154.8 & 63.0 & 199.1 \\
\hline & & DA & 137.9 & 52.4 & 60.9 & 2116 & 18.4 & 110.8 & 41.3 & 196.9 \\
\hline & & BDA & 134.3 & 45.0 & 52.3 & 2116 & 0.0 & 95.2 & 35.5 & 169.2 \\
\hline \multirow[t]{3}{*}{$2 a$} & \multirow[t]{3}{*}{3200} & JM & 50.8 & 37.9 & 40.5 & 1860 & 0.0 & 70.6 & 23.2 & 305.1 \\
\hline & & DA & 48.8 & 45.0 & 48.7 & 2116 & 0.0 & 95.2 & 33.9 & 305.1 \\
\hline & & BDA & 48.8 & 45.0 & 48.7 & 2116 & 0.0 & 95.2 & 33.9 & 305.1 \\
\hline \multirow[t]{3}{*}{$2 b$} & \multirow[t]{3}{*}{4150} & JM & 92.2 & 49.0 & 53.4 & 2267 & 0.0 & 111.0 & 41.0 & 305.1 \\
\hline & & DA & 91.5 & 45.0 & 48.7 & 2116 & 0.0 & 95.2 & 33.9 & 305.1 \\
\hline & & BDA & 91.5 & 45.0 & 48.7 & 2116 & 0.0 & 95.2 & 33.9 & 305.1 \\
\hline \multirow[t]{3}{*}{$2 c$} & \multirow[t]{3}{*}{5100} & JM & 143.5 & 58.8 & 65.3 & 2658 & 0.0 & 156.2 & 62.1 & 305.1 \\
\hline & & DA & 134.3 & 45.0 & 48.7 & 2116 & 0.0 & 95.2 & 33.9 & 305.1 \\
\hline & & BDA & 134.3 & 45.0 & 48.7 & 2116 & 0.0 & 95.2 & 33.9 & 305.1 \\
\hline
\end{tabular}


Table D2

Solutions of base-case scenario for case 3 .

\begin{tabular}{|c|c|c|c|c|c|c|c|c|c|c|}
\hline $\begin{array}{l}\text { Case } \\
\mathrm{Nb} .\end{array}$ & $\begin{array}{l}\beta \\
(\$)\end{array}$ & Mechanism & $\begin{array}{l}\text { Objective } \\
\left(\$ \times 10^{6}\right)\end{array}$ & $\begin{array}{l}\text { Exp. sales } \\
\left(\times 10^{3} \text { vehicles }\right)\end{array}$ & $\begin{array}{l}Q \\
\left(\times 10^{3} \text { vehicles }\right)\end{array}$ & $\begin{array}{l}r \\
(\$)\end{array}$ & $\begin{array}{l}B_{d} \\
\left(\$ \times 10^{6}\right)\end{array}$ & $\begin{array}{l}B_{r} \\
\left(\$ \times 10^{6}\right)\end{array}$ & $\begin{array}{l}E[P(Q)] \\
\left(\$ \times 10^{6}\right)\end{array}$ & $\begin{array}{l}\mu\left(B_{d}\right) \\
\left(\times 10^{3} \text { vehicles }\right)\end{array}$ \\
\hline \multirow[t]{3}{*}{$3 a$} & \multirow[t]{3}{*}{3200} & JM & 72.9 & 54.2 & 56.3 & 1324 & 28.7 & 71.7 & 26.2 & 154.6 \\
\hline & & DA & 50.6 & 58.6 & 64.0 & 2116 & 13.0 & 124.1 & 62.9 & 127.1 \\
\hline & & $\mathrm{BDA}$ & 48.8 & 45.0 & 49.1 & 2116 & 0.0 & 95.2 & 48.3 & 97.5 \\
\hline \multirow[t]{3}{*}{$3 b$} & \multirow[t]{3}{*}{4150} & JM & 134.5 & 74.6 & 78.7 & 1569 & 58.0 & 117.0 & 49.6 & 190.5 \\
\hline & & DA & 120.9 & 85.3 & 93.0 & 2116 & 52.5 & 180.4 & 91.4 & 184.8 \\
\hline & & BDA & 91.5 & 45.0 & 49.1 & 2116 & 0.0 & 95.2 & 48.3 & 97.5 \\
\hline \multirow[t]{3}{*}{$3 c$} & \multirow[t]{3}{*}{5100} & JM & 213.2 & 90.4 & 96.8 & 1806 & 84.5 & 163.2 & 76.0 & 212.7 \\
\hline & & DA & 208.4 & 97.7 & 106.5 & 2116 & 83.0 & 206.6 & 104.7 & 211.7 \\
\hline & & BDA & 134.3 & 45.0 & 49.1 & 2116 & 0.0 & 95.2 & 48.3 & 97.5 \\
\hline \multirow[t]{3}{*}{$3 d$} & \multirow[t]{3}{*}{3200} & JM & 62.1 & 40.3 & 42.4 & 1413 & 9.9 & 57.0 & 21.1 & 139.3 \\
\hline & & DA & 48.8 & 45.0 & 49.6 & 2116 & 0.0 & 95.2 & 46.0 & 118.1 \\
\hline & & BDA & 48.8 & 45.0 & 49.6 & 2116 & 0.0 & 95.2 & 46.0 & 118.1 \\
\hline \multirow[t]{3}{*}{$3 e$} & \multirow[t]{3}{*}{4150} & JM & 109.9 & 59.4 & 63.7 & 1689 & 36.3 & 100.3 & 42.7 & 180.0 \\
\hline & & DA & 103.3 & 67.1 & 74.1 & 2116 & 33.3 & 142.0 & 68.7 & 176.2 \\
\hline & & BDA & 91.5 & 45.0 & 49.6 & 2116 & 0.0 & 95.2 & 46.0 & 118.1 \\
\hline \multirow[t]{3}{*}{$3 \mathrm{f}$} & \multirow[t]{3}{*}{5100} & JM & 173.7 & 74.4 & 81.3 & 1956 & 60.3 & 145.6 & 67.7 & 204.9 \\
\hline & & DA & 172.7 & 78.0 & 86.0 & 2116 & 59.9 & 165.0 & 79.7 & 204.6 \\
\hline & & $\mathrm{BDA}$ & 134.3 & 45.0 & 49.6 & 2116 & 0.0 & 95.2 & 46.0 & 118.1 \\
\hline \multirow[t]{3}{*}{$3 \mathrm{~g}$} & \multirow[t]{3}{*}{3200} & $\mathrm{JM}$ & 58.9 & 34.3 & 36.4 & 1483 & 0.0 & 50.9 & 19.0 & 141.2 \\
\hline & & DA & 48.8 & 45.0 & 50.1 & 2116 & 0.0 & 95.2 & 44.2 & 141.2 \\
\hline & & BDA & 48.8 & 45.0 & 50.1 & 2116 & 0.0 & 95.2 & 44.2 & 141.2 \\
\hline \multirow[t]{3}{*}{$3 \mathrm{~h}$} & \multirow[t]{3}{*}{4150} & $\mathrm{JM}$ & 97.5 & 48.6 & 52.9 & 1785 & 17.6 & 86.8 & 36.8 & 172.4 \\
\hline & & DA & 94.2 & 54.2 & 60.3 & 2116 & 15.9 & 114.6 & 53.2 & 169.9 \\
\hline & & BDA & 91.5 & 45.0 & 50.1 & 2116 & 0.0 & 95.2 & 44.2 & 141.2 \\
\hline \multirow[t]{3}{*}{$3 i$} & \multirow[t]{3}{*}{5100} & $\mathrm{JM}$ & 150.6 & 62.8 & 69.7 & 2077 & 39.2 & 130.4 & 60.0 & 199.5 \\
\hline & & DA & 150.6 & 63.6 & 70.8 & 2116 & 39.2 & 134.5 & 62.5 & 199.5 \\
\hline & & BDA & 134.3 & 45.0 & 50.1 & 2116 & 0.0 & 95.2 & 44.2 & 141.2 \\
\hline
\end{tabular}

Table D3

Solutions of base-case scenario for case 4 .

\begin{tabular}{|c|c|c|c|c|c|c|c|c|c|c|}
\hline $\begin{array}{l}\text { Case } \\
\mathrm{Nb} \text {. }\end{array}$ & $\begin{array}{l}\beta \\
(\$)\end{array}$ & Mechanism & $\begin{array}{l}\text { Objective } \\
\left(\$ \times 10^{6}\right)\end{array}$ & $\begin{array}{l}\text { Exp. sales } \\
\left(\times 10^{3} \text { vehicles }\right)\end{array}$ & $\begin{array}{l}Q \\
\left(\times 10^{3} \text { vehicles }\right)\end{array}$ & $\begin{array}{l}r \\
(\$)\end{array}$ & $\begin{array}{l}B_{d} \\
\left(\$ \times 10^{6}\right)\end{array}$ & $\begin{array}{l}B_{r} \\
\left(\$ \times 10^{6}\right)\end{array}$ & $\begin{array}{l}E[P(Q)] \\
\left(\$ \times 10^{6}\right)\end{array}$ & $\begin{array}{l}\mu\left(B_{d}\right) \\
\left(\times 10^{3} \text { vehicles }\right)\end{array}$ \\
\hline \multirow[t]{3}{*}{$4 a$} & \multirow[t]{3}{*}{3200} & JM & 65.2 & 38.4 & 39.1 & 1299 & 7.8 & 49.9 & 18.1 & 142.0 \\
\hline & & DA & 48.8 & 45.0 & 46.9 & 2116 & 0.0 & 95.2 & 48.7 & 125.5 \\
\hline & & BDA & 48.8 & 45.0 & 46.9 & 2116 & 0.0 & 95.2 & 48.7 & 125.5 \\
\hline \multirow[t]{3}{*}{$4 b$} & \multirow[t]{3}{*}{4150} & JM & 109.6 & 54.5 & 55.8 & 1549 & 32.1 & 84.4 & 35.9 & 180.0 \\
\hline & & DA & 99.5 & 62.4 & 65.0 & 2116 & 27.4 & 132.0 & 67.4 & 173.8 \\
\hline & & BDA & 91.5 & 45.0 & 46.9 & 2116 & 0.0 & 95.2 & 48.7 & 125.5 \\
\hline \multirow[t]{3}{*}{$4 c$} & \multirow[t]{3}{*}{5100} & JM & 167.6 & 67.1 & 69.3 & 1799 & 54.0 & 120.8 & 56.8 & 203.8 \\
\hline & & DA & 164.1 & 72.7 & 75.8 & 2116 & 52.9 & 153.9 & 78.6 & 202.7 \\
\hline & & BDA & 134.3 & 45.0 & 46.9 & 2116 & 0.0 & 95.2 & 48.7 & 125.5 \\
\hline \multirow[t]{3}{*}{$4 d$} & \multirow[t]{3}{*}{3200} & JM & 61.4 & 33.9 & 34.7 & 1388 & 0.0 & 47.0 & 17.5 & 159.5 \\
\hline & & DA & 48.8 & 45.0 & 47.2 & 2116 & 0.0 & 95.2 & 46.5 & 159.5 \\
\hline & & BDA & 48.8 & 45.0 & 47.2 & 2116 & 0.0 & 95.2 & 46.5 & 159.5 \\
\hline \multirow[t]{3}{*}{$4 e$} & \multirow[t]{3}{*}{4150} & JM & 96.5 & 41.6 & 43.0 & 1672 & 6.7 & 69.6 & 29.9 & 171.4 \\
\hline & & DA & 91.7 & 47.2 & 49.5 & 2116 & 4.3 & 99.9 & 48.8 & 167.3 \\
\hline & & BDA & 91.5 & 45.0 & 47.2 & 2116 & 0.0 & 95.2 & 46.5 & 159.5 \\
\hline \multirow[t]{3}{*}{$4 \mathrm{f}$} & \multirow[t]{3}{*}{5100} & JM & 141.7 & 53.1 & 55.4 & 1955 & 25.4 & 103.9 & 48.9 & 197.7 \\
\hline & & DA & 141.0 & 55.7 & 58.4 & 2116 & 25.2 & 117.8 & 57.5 & 197.4 \\
\hline & & BDA & 134.3 & 45.0 & 47.2 & 2116 & 0.0 & 95.2 & 46.5 & 159.5 \\
\hline \multirow[t]{3}{*}{$4 \mathrm{~g}$} & \multirow[t]{3}{*}{3200} & JM & 59.2 & 34.0 & 35.0 & 1459 & 0.0 & 49.6 & 18.6 & 199.0 \\
\hline & & DA & 48.8 & 45.0 & 47.4 & 2116 & 0.0 & 95.2 & 44.8 & 199.0 \\
\hline & & BDA & 48.8 & 45.0 & 47.4 & 2116 & 0.0 & 95.2 & 44.8 & 199.0 \\
\hline \multirow[t]{3}{*}{$4 h$} & \multirow[t]{3}{*}{4150} & JM & 94.3 & 39.6 & 41.2 & 1770 & 0.0 & 70.1 & 30.1 & 199.0 \\
\hline & & DA & 91.5 & 45.0 & 47.4 & 2116 & 0.0 & 95.2 & 44.8 & 199.0 \\
\hline & & BDA & 91.5 & 45.0 & 47.4 & 2116 & 0.0 & 95.2 & 44.8 & 199.0 \\
\hline \multirow[t]{3}{*}{$4 i$} & \multirow[t]{3}{*}{5100} & JM & 134.3 & 44.5 & 46.8 & 2080 & 0.0 & 92.5 & 43.2 & 199.0 \\
\hline & & DA & 134.3 & 45.0 & 47.4 & 2116 & 0.0 & 95.2 & 44.8 & 199.0 \\
\hline & & BDA & 134.3 & 45.0 & 47.4 & 2116 & 0.0 & 95.2 & 44.8 & 199.0 \\
\hline
\end{tabular}


Table D4

Solutions of medium-term scenario for cases 5 and 6 .

\begin{tabular}{|c|c|c|c|c|c|c|c|c|c|c|}
\hline $\begin{array}{l}\text { Case } \\
\mathrm{Nb} .\end{array}$ & $\begin{array}{l}\beta \\
(\$)\end{array}$ & Mechanism & $\begin{array}{l}\text { Objective } \\
\left(\$ \times 10^{6}\right)\end{array}$ & $\begin{array}{l}\text { Exp. sales } \\
\left(\times 10^{3} \text { vehicles }\right)\end{array}$ & $\begin{array}{l}Q \\
\left(\times 10^{3} \text { vehicles }\right)\end{array}$ & $\begin{array}{l}r \\
(\$)\end{array}$ & $\begin{array}{l}B_{d} \\
\left(\$ \times 10^{6}\right)\end{array}$ & $\begin{array}{l}B_{r} \\
\left(\$ \times 10^{6}\right)\end{array}$ & $\begin{array}{l}E[P(Q)] \\
\left(\$ \times 10^{6}\right)\end{array}$ & $\begin{array}{l}\mu\left(B_{d}\right) \\
\left(\times 10^{3} \text { vehicles }\right)\end{array}$ \\
\hline \multirow[t]{3}{*}{$5 a$} & \multirow[t]{3}{*}{3200} & JM & 177.7 & 150.0 & 169.2 & 1789 & 33.9 & 268.3 & 89.5 & 686.7 \\
\hline & & DA & 164.2 & 178.9 & 208.0 & 2116 & 29.7 & 378.6 & 141.1 & 672.6 \\
\hline & & BDA & 154.8 & 142.8 & 166.0 & 2116 & 0.0 & 302.2 & 112.6 & 536.9 \\
\hline \multirow[t]{3}{*}{$5 b$} & \multirow[t]{3}{*}{4150} & JM & 351.1 & 212.8 & 248.1 & 2147 & 75.1 & 457.1 & 171.8 & 787.6 \\
\hline & & DA & 351.0 & 209.5 & 243.5 & 2116 & 75.1 & 443.2 & 165.2 & 787.5 \\
\hline & & BDA & 290.5 & 142.8 & 166.0 & 2116 & 0.0 & 302.2 & 112.6 & 536.9 \\
\hline \multirow[t]{3}{*}{$5 c$} & \multirow[t]{3}{*}{5100} & JM & 578.6 & 264.4 & 317.2 & 2484 & 113.3 & 656.7 & 267.3 & 844.9 \\
\hline & & DA & 557.4 & 223.7 & 260.1 & 2116 & 110.1 & 473.4 & 176.4 & 841.0 \\
\hline & & BDA & 426.2 & 142.8 & 166.0 & 2116 & 0.0 & 302.2 & 112.6 & 536.9 \\
\hline \multirow[t]{3}{*}{$6 a$} & \multirow[t]{3}{*}{3200} & JM & 165.7 & 123.6 & 132.0 & 1860 & 0.0 & 229.9 & 75.7 & 994.2 \\
\hline & & DA & 158.9 & 146.6 & 158.6 & 2116 & 0.0 & 310.2 & 110.4 & 994.2 \\
\hline & & BDA & 158.9 & 146.6 & 158.6 & 2116 & 0.0 & 310.2 & 110.4 & 994.2 \\
\hline \multirow[t]{3}{*}{$6 b$} & \multirow[t]{3}{*}{4150} & JM & 300.6 & 159.6 & 174.0 & 2267 & 0.0 & 361.8 & 133.5 & 994.2 \\
\hline & & DA & 298.2 & 146.6 & 158.6 & 2116 & 0.0 & 310.2 & 110.4 & 994.2 \\
\hline & & BDA & 298.2 & 146.6 & 158.6 & 2116 & 0.0 & 310.2 & 110.4 & 994.2 \\
\hline \multirow[t]{3}{*}{$6 c$} & \multirow[t]{3}{*}{5100} & JM & 467.6 & 191.5 & 212.7 & 2658 & 0.0 & 508.9 & 202.2 & 994.2 \\
\hline & & DA & 437.5 & 146.6 & 158.6 & 2116 & 0.0 & 310.2 & 110.4 & 994.2 \\
\hline & & BDA & 437.5 & 146.6 & 158.6 & 2116 & 0.0 & 310.2 & 110.4 & 994.2 \\
\hline
\end{tabular}

Table D5

Solutions of medium-term scenario for case 7.

\begin{tabular}{|c|c|c|c|c|c|c|c|c|c|c|}
\hline $\begin{array}{l}\text { Case } \\
\mathrm{Nb} .\end{array}$ & $\begin{array}{l}\beta \\
(\$)\end{array}$ & Mechanism & $\begin{array}{l}\text { Objective } \\
\left(\$ \times 10^{6}\right)\end{array}$ & $\begin{array}{l}\text { Exp. sales } \\
\left(\times 10^{3} \text { vehicles }\right)\end{array}$ & $\begin{array}{l}Q \\
\left(\times 10^{3} \text { vehicles }\right)\end{array}$ & $\begin{array}{l}r \\
(\$)\end{array}$ & $\begin{array}{l}B_{d} \\
\left(\$ \times 10^{6}\right)\end{array}$ & $\begin{array}{l}B_{r} \\
\left(\$ \times 10^{6}\right)\end{array}$ & $\begin{array}{l}E[P(Q)] \\
\left(\$ \times 10^{6}\right)\end{array}$ & $\begin{array}{l}\mu\left(B_{d}\right) \\
\left(\times 10^{3} \text { vehicles }\right)\end{array}$ \\
\hline \multirow[t]{3}{*}{$7 a$} & \multirow[t]{3}{*}{3200} & JM & 373.5 & 268.0 & 278.6 & 1324 & 129.3 & 354.9 & 129.5 & 764.7 \\
\hline & & DA & 256.7 & 330.2 & 360.1 & 2116 & 101.3 & 698.7 & 354.2 & 715.8 \\
\hline & & BDA & 145.9 & 134.6 & 146.8 & 2116 & 0.0 & 284.9 & 144.4 & 291.8 \\
\hline \multirow[t]{3}{*}{$7 b$} & \multirow[t]{3}{*}{4150} & JM & 656.2 & 324.5 & 342.4 & 1569 & 181.4 & 509.1 & 215.7 & 828.6 \\
\hline & & DA & 596.5 & 377.6 & 411.8 & 2116 & 171.6 & 799.1 & 405.0 & 818.6 \\
\hline & & BDA & 273.8 & 134.6 & 146.8 & 2116 & 0.0 & 284.9 & 144.4 & 291.8 \\
\hline \multirow[t]{3}{*}{ 7c } & \multirow[t]{3}{*}{5100} & JM & 986.3 & 368.9 & 394.9 & 1806 & 228.5 & 666.3 & 310.4 & 868.2 \\
\hline & & DA & 966.7 & 399.7 & 435.9 & 2116 & 226.0 & 845.8 & 428.7 & 866.4 \\
\hline & & BDA & 401.8 & 134.6 & 146.8 & 2116 & 0.0 & 284.9 & 144.4 & 291.8 \\
\hline \multirow[t]{3}{*}{$7 d$} & \multirow[t]{3}{*}{3200} & JM & 285.7 & 213.4 & 224.4 & 1413 & 95.8 & 301.5 & 111.9 & 737.6 \\
\hline & & DA & 210.9 & 264.6 & 291.9 & 2116 & 75.9 & 559.8 & 270.6 & 694.5 \\
\hline & & BDA & 149.1 & 137.5 & 151.7 & 2116 & 0.0 & 291.0 & 140.7 & 361.0 \\
\hline \multirow[t]{3}{*}{ 7e } & \multirow[t]{3}{*}{4150} & JM & 515.1 & 267.3 & 286.4 & 1689 & 142.7 & 451.3 & 192.3 & 810.0 \\
\hline & & DA & 485.0 & 306.0 & 337.6 & 2116 & 137.4 & 647.5 & 313.0 & 803.2 \\
\hline & & BDA & 279.7 & 137.5 & 151.7 & 2116 & 0.0 & 291.0 & 140.7 & 361.0 \\
\hline \multirow[t]{3}{*}{$7 f$} & \multirow[t]{3}{*}{5100} & JM & 790.1 & 310.3 & 338.8 & 1956 & 185.5 & 607.2 & 282.2 & 854.4 \\
\hline & & DA & 785.8 & 325.3 & 358.8 & 2116 & 184.9 & 688.3 & 332.7 & 853.9 \\
\hline & & BDA & 410.4 & 137.5 & 151.7 & 2116 & 0.0 & 291.0 & 140.7 & 361.0 \\
\hline \multirow[t]{3}{*}{$7 \mathrm{~g}$} & \multirow[t]{3}{*}{3200} & JM & 231.7 & 174.2 & 185.1 & 1483 & 67.4 & 258.5 & 96.3 & 716.9 \\
\hline & & DA & 181.5 & 216.4 & 240.9 & 2116 & 53.0 & 457.9 & 212.7 & 679.0 \\
\hline & & BDA & 151.5 & 139.8 & 155.6 & 2116 & 0.0 & 295.8 & 137.4 & 438.6 \\
\hline \multirow[t]{3}{*}{$7 \mathrm{~h}$} & \multirow[t]{3}{*}{4150} & JM & 422.1 & 224.7 & 244.2 & 1785 & 109.4 & 401.0 & 170.1 & 796.4 \\
\hline & & DA & 406.9 & 252.4 & 281.0 & 2116 & 106.5 & 534.1 & 248.1 & 792.1 \\
\hline & & BDA & 284.3 & 139.8 & 155.6 & 2116 & 0.0 & 295.8 & 137.4 & 438.6 \\
\hline \multirow[t]{3}{*}{$7 i$} & \multirow[t]{3}{*}{5100} & JM & 655.7 & 265.8 & 295.1 & 2077 & 147.9 & 552.0 & 254.1 & 844.8 \\
\hline & & DA & 655.5 & 269.2 & 299.7 & 2116 & 147.9 & 569.6 & 264.6 & 844.8 \\
\hline & & BDA & 417.1 & 139.8 & 155.6 & 2116 & 0.0 & 295.8 & 137.4 & 438.6 \\
\hline
\end{tabular}


Table D6

Solutions of medium-term scenario for case 8 .

\begin{tabular}{|c|c|c|c|c|c|c|c|c|c|c|}
\hline $\begin{array}{l}\text { Case } \\
\mathrm{Nb} .\end{array}$ & $\begin{array}{l}\beta \\
(\$)\end{array}$ & Mechanism & $\begin{array}{l}\text { Objective } \\
\left(\$ \times 10^{6}\right)\end{array}$ & $\begin{array}{l}\text { Exp. sales } \\
\left(\times 10^{3} \text { vehicles }\right)\end{array}$ & $\begin{array}{l}Q \\
\left(\times 10^{3} \text { vehicles }\right)\end{array}$ & $\begin{array}{l}r \\
(\$)\end{array}$ & $\begin{array}{l}B_{d} \\
\left(\$ \times 10^{6}\right)\end{array}$ & $\begin{array}{l}B_{r} \\
\left(\$ \times 10^{6}\right)\end{array}$ & $\begin{array}{l}\mathrm{E}[\mathrm{P}(\mathrm{Q})] \\
\left(\$ \times 10^{6}\right)\end{array}$ & $\begin{array}{l}\mu\left(B_{d}\right) \\
\left(\times 10^{3} \text { vehicles }\right)\end{array}$ \\
\hline \multirow[t]{3}{*}{$8 a$} & \multirow[t]{3}{*}{3200} & JM & 289.7 & 200.8 & 204.2 & 1299 & 92.1 & 260.7 & 94.8 & 742.2 \\
\hline & & DA & 199.6 & 247.0 & 257.4 & 2116 & 68.1 & 522.7 & 267.1 & 688.6 \\
\hline & & BDA & 150.0 & 138.3 & 144.2 & 2116 & 0.0 & 292.7 & 149.6 & 385.6 \\
\hline \multirow[t]{3}{*}{$8 b$} & \multirow[t]{3}{*}{4150} & JM & 502.3 & 245.1 & 251.1 & 1549 & 135.2 & 379.6 & 161.6 & 810.0 \\
\hline & & DA & 456.1 & 286.6 & 298.7 & 2116 & 126.9 & 606.5 & 309.9 & 799.0 \\
\hline & & BDA & 281.4 & 138.3 & 144.2 & 2116 & 0.0 & 292.7 & 149.6 & 385.6 \\
\hline \multirow[t]{3}{*}{$8 c$} & \multirow[t]{3}{*}{5100} & JM & 752.6 & 280.8 & 289.9 & 1799 & 174.4 & 505.2 & 237.6 & 852.4 \\
\hline & & DA & 738.0 & 305.1 & 317.9 & 2116 & 172.3 & 645.5 & 329.9 & 850.4 \\
\hline & & BDA & 412.8 & 138.3 & 144.2 & 2116 & 0.0 & 292.7 & 149.6 & 385.6 \\
\hline \multirow[t]{3}{*}{$8 d$} & \multirow[t]{3}{*}{3200} & JM & 223.4 & 152.9 & 156.3 & 1388 & 53.7 & 212.1 & 78.9 & 719.1 \\
\hline & & DA & 168.0 & 189.7 & 198.8 & 2116 & 37.7 & 401.5 & 196.0 & 672.5 \\
\hline & & BDA & 152.9 & 141.1 & 147.9 & 2116 & 0.0 & 298.5 & 145.8 & 500.1 \\
\hline \multirow[t]{3}{*}{$8 \mathrm{e}$} & \multirow[t]{3}{*}{4150} & JM & 388.4 & 193.1 & 199.3 & 1672 & 90.1 & 322.8 & 138.8 & 794.7 \\
\hline & & DA & 366.1 & 222.2 & 232.8 & 2116 & 85.8 & 470.1 & 229.5 & 787.4 \\
\hline & & BDA & 287.0 & 141.1 & 147.9 & 2116 & 0.0 & 298.5 & 145.8 & 500.1 \\
\hline \multirow[t]{3}{*}{$8 \mathrm{f}$} & \multirow[t]{3}{*}{5100} & JM & 588.0 & 226.2 & 235.8 & 1955 & 123.4 & 442.4 & 208.1 & 841.5 \\
\hline & & DA & 585.0 & 237.3 & 248.6 & 2116 & 123.0 & 502.0 & 245.1 & 840.9 \\
\hline & & BDA & 421.0 & 141.1 & 147.9 & 2116 & 0.0 & 298.5 & 145.8 & 500.1 \\
\hline \multirow[t]{3}{*}{$8 g$} & \multirow[t]{3}{*}{3200} & JM & 192.7 & 122.3 & 125.6 & 1459 & 20.2 & 178.4 & 66.9 & 714.9 \\
\hline & & DA & 156.1 & 152.8 & 160.8 & 2116 & 9.5 & 323.3 & 152.0 & 675.5 \\
\hline & & BDA & 155.1 & 143.1 & 150.6 & 2116 & 0.0 & 302.8 & 142.3 & 632.7 \\
\hline \multirow[t]{3}{*}{$8 \mathrm{~h}$} & \multirow[t]{3}{*}{4150} & JM & 326.5 & 158.2 & 164.4 & 1770 & 49.9 & 280.0 & 120.1 & 794.1 \\
\hline & & DA & 315.4 & 178.6 & 188.0 & 2116 & 47.8 & 377.9 & 177.6 & 789.6 \\
\hline & & BDA & 291.1 & 143.1 & 150.6 & 2116 & 0.0 & 302.8 & 142.3 & 632.7 \\
\hline \multirow[t]{3}{*}{$8 \mathrm{i}$} & \multirow[t]{3}{*}{5100} & JM & 491.5 & 188.4 & 198.1 & 2080 & 77.4 & 392.0 & 182.8 & 842.8 \\
\hline & & DA & 491.3 & 190.6 & 200.6 & 2116 & 77.4 & 403.3 & 189.6 & 842.7 \\
\hline & & BDA & 427.0 & 143.1 & 150.6 & 2116 & 0.0 & 302.8 & 142.3 & 632.7 \\
\hline
\end{tabular}

Table D7

Solutions of long-term scenario for cases 9 and 10 .

\begin{tabular}{|c|c|c|c|c|c|c|c|c|c|c|}
\hline $\begin{array}{l}\text { Case } \\
\mathrm{Nb} .\end{array}$ & $\begin{array}{l}\beta \\
(\$)\end{array}$ & Mechanism & $\begin{array}{l}\text { Objective } \\
\left(\$ \times 10^{6}\right)\end{array}$ & $\begin{array}{l}\text { Exp. sales } \\
\left(\times 10^{3} \text { vehicles }\right)\end{array}$ & $\begin{array}{l}Q \\
\left(\times 10^{3} \text { vehicles }\right)\end{array}$ & $\begin{array}{l}r \\
(\$)\end{array}$ & $\begin{array}{l}B_{d} \\
\left(\$ \times 10^{6}\right)\end{array}$ & $\begin{array}{l}B_{r} \\
\left(\$ \times 10^{6}\right)\end{array}$ & $\begin{array}{l}\mathrm{E}[\mathrm{P}(\mathrm{Q})] \\
\left(\$ \times 10^{6}\right)\end{array}$ & $\begin{array}{l}\mu\left(B_{d}\right) \\
\left(\times 10^{3} \text { vehicles }\right)\end{array}$ \\
\hline \multirow[t]{3}{*}{$9 a$} & \multirow[t]{3}{*}{3200} & JM & 783.2 & 639.6 & 721.6 & 1789 & 119.3 & 1144.1 & 381.6 & 2927.8 \\
\hline & & DA & 725.1 & 772.7 & 898.3 & 2116 & 112.5 & 1635.0 & 609.2 & 2904.8 \\
\hline & & $\mathrm{BDA}$ & 588.3 & 542.7 & 630.9 & 2116 & 0.0 & 1148.3 & 427.9 & 2040.2 \\
\hline \multirow[t]{3}{*}{$9 b$} & \multirow[t]{3}{*}{4150} & JM & 1487.3 & 835.9 & 974.5 & 2147 & 186.7 & 1795.0 & 674.7 & 3093.0 \\
\hline & & DA & 1486.7 & 822.7 & 956.5 & 2116 & 186.7 & 1740.9 & 648.7 & 3092.9 \\
\hline & & $\mathrm{BDA}$ & 1103.8 & 542.7 & 630.9 & 2116 & 0.0 & 1148.3 & 427.9 & 2040.2 \\
\hline \multirow[t]{3}{*}{$9 c$} & \multirow[t]{3}{*}{5100} & JM & 2360.4 & 997.4 & 1196.3 & 2484 & 249.2 & 2477.2 & 1008.1 & 3186.9 \\
\hline & & DA & 2280.4 & 846.0 & 983.6 & 2116 & 244.1 & 1790.2 & 667.1 & 3180.6 \\
\hline & & BDA & 1619.4 & 542.7 & 630.9 & 2116 & 0.0 & 1148.3 & 427.9 & 2040.2 \\
\hline \multirow[t]{3}{*}{$10 a$} & \multirow[t]{3}{*}{3200} & JM & 554.6 & 413.8 & 441.9 & 1860 & 0.0 & 769.5 & 253.6 & 3328.1 \\
\hline & & DA & 532.1 & 490.8 & 531.0 & 2116 & 0.0 & 1038.6 & 369.6 & 3328.1 \\
\hline & & $\mathrm{BDA}$ & 532.1 & 490.8 & 531.0 & 2116 & 0.0 & 1038.6 & 369.6 & 3328.1 \\
\hline \multirow[t]{3}{*}{$10 \mathrm{~b}$} & \multirow[t]{3}{*}{4150} & JM & 1006.2 & 534.3 & 582.4 & 2267 & 0.0 & 1211.2 & 446.9 & 3328.1 \\
\hline & & DA & 998.4 & 490.8 & 531.0 & 2116.0 & 0.0 & 1038.6 & 369.6 & 3328.1 \\
\hline & & BDA & 998.4 & 490.8 & 531.0 & 2116 & 0.0 & 1038.6 & 369.6 & 3328.1 \\
\hline \multirow[t]{3}{*}{$10 c$} & \multirow[t]{3}{*}{5100} & JM & 1567.0 & 646.7 & 718.3 & 2658 & 12.3 & 1718.8 & 683.0 & 3357.6 \\
\hline & & DA & 1465.5 & 494.1 & 534.5 & 2116 & 8.8 & 1045.4 & 372.0 & 3349.9 \\
\hline & & $\mathrm{BDA}$ & 1464.6 & 490.8 & 531.0 & 2116 & 0.0 & 1038.6 & 369.6 & 3328.1 \\
\hline
\end{tabular}


Table D8

Solutions of long-term scenario for case 11 .

\begin{tabular}{|c|c|c|c|c|c|c|c|c|c|c|}
\hline $\begin{array}{l}\text { Case } \\
\mathrm{Nb} .\end{array}$ & $\begin{array}{l}\beta \\
(\$)\end{array}$ & Mechanism & $\begin{array}{l}\text { Objective } \\
\left(\$ \times 10^{6}\right)\end{array}$ & $\begin{array}{l}\text { Exp. sales } \\
\left(\times 10^{3} \text { vehicles }\right)\end{array}$ & $\begin{array}{l}Q \\
\left(\times 10^{3} \text { vehicles }\right)\end{array}$ & $\begin{array}{l}r \\
(\$)\end{array}$ & $\begin{array}{l}B_{d} \\
\left(\$ \times 10^{6}\right)\end{array}$ & $\begin{array}{l}B_{r} \\
\left(\$ \times 10^{6}\right)\end{array}$ & $\begin{array}{l}\mathrm{E}[\mathrm{P}(\mathrm{Q})] \\
\left(\$ \times 10^{6}\right)\end{array}$ & $\begin{array}{l}\mu\left(B_{d}\right) \\
\left(\times 10^{3} \text { vehicles }\right)\end{array}$ \\
\hline \multirow[t]{3}{*}{$11 \mathrm{a}$} & \multirow[t]{3}{*}{3200} & JM & 1733.9 & 1071.1 & 1113.1 & 1324 & 275.5 & 1418.0 & 517.4 & 3055.6 \\
\hline & & DA & 1258.4 & 1372.7 & 1497.1 & 2116 & 229.6 & 2904.6 & 1472.3 & 2975.5 \\
\hline & & BDA & 675.0 & 622.7 & 679.1 & 2116 & 0.0 & 1317.6 & 667.9 & 1349.8 \\
\hline \multirow[t]{3}{*}{$11 b$} & \multirow[t]{3}{*}{4150} & JM & 2833.9 & 1237.6 & 1306.0 & 1569 & 360.8 & 1941.5 & 822.7 & 3160.3 \\
\hline & & DA & 2605.2 & 1450.3 & 1581.7 & 2116 & 344.8 & 3068.9 & 1555.5 & 3143.8 \\
\hline & & BDA & 1266.6 & 622.7 & 679.1 & 2116 & 0.0 & 1317.6 & 667.9 & 1349.8 \\
\hline \multirow[t]{3}{*}{$11 \mathrm{c}$} & \multirow[t]{3}{*}{5100} & JM & 4074.7 & 1370.1 & 1467.0 & 1806 & 438.0 & 2475.0 & 1152.8 & 3225.1 \\
\hline & & DA & 4001.9 & 1486.5 & 1621.1 & 2116 & 433.8 & 3145.4 & 1594.3 & 3222.2 \\
\hline & & BDA & 1858.1 & 622.7 & 679.1 & 2116 & 0.0 & 1317.6 & 667.9 & 1349.8 \\
\hline \multirow[t]{3}{*}{$11 d$} & \multirow[t]{3}{*}{3200} & JM & 1336.8 & 871.3 & 916.0 & 1413 & 220.6 & 1230.8 & 456.8 & 3011.1 \\
\hline & & DA & 1026.3 & 1120.2 & 1235.8 & 2116 & 188.0 & 2370.4 & 1145.9 & 2940.6 \\
\hline & & BDA & 637.9 & 588.4 & 649.1 & 2116 & 0.0 & 1245.1 & 601.9 & 1544.6 \\
\hline \multirow[t]{3}{*}{$11 \mathrm{e}$} & \multirow[t]{3}{*}{4150} & JM & 2244.2 & 1032.7 & 1106.8 & 1689 & 297.5 & 1743.9 & 743.0 & 3129.7 \\
\hline & & DA & 2127.9 & 1188.1 & 1310.6 & 2116 & 288.7 & 2514.0 & 1215.3 & 3118.7 \\
\hline & & BDA & 1196.9 & 588.4 & 649.1 & 2116 & 0.0 & 1245.1 & 601.9 & 1544.6 \\
\hline \multirow[t]{3}{*}{$11 \mathrm{f}$} & \multirow[t]{3}{*}{5100} & JM & 3289.2 & 1163.3 & 1269.7 & 1956 & 367.5 & 2275.9 & 1057.8 & 3202.5 \\
\hline & & DA & 3273.0 & 1219.7 & 1345.5 & 2116 & 366.5 & 2580.8 & 1247.6 & 3201.6 \\
\hline & & BDA & 1755.9 & 588.4 & 649.1 & 2116 & 0.0 & 1245.1 & 601.9 & 1544.6 \\
\hline \multirow[t]{3}{*}{$11 \mathrm{~g}$} & \multirow[t]{3}{*}{3200} & JM & 1068.1 & 723.6 & 768.5 & 1483 & 174.2 & 1073.4 & 399.9 & 2977.3 \\
\hline & & DA & 856.5 & 929.0 & 1034.3 & 2116 & 150.6 & 1965.8 & 913.3 & 2915.2 \\
\hline & & BDA & 609.1 & 561.9 & 625.5 & 2116 & 0.0 & 1188.9 & 552.4 & 1763.1 \\
\hline \multirow[t]{3}{*}{$11 \mathrm{~h}$} & \multirow[t]{3}{*}{4150} & JM & 1830.9 & 876.8 & 952.7 & 1785 & 242.9 & 1564.8 & 663.8 & 3107.6 \\
\hline & & DA & 1771.6 & 988.1 & 1100.0 & 2116 & 238.2 & 2090.7 & 971.3 & 3100.4 \\
\hline & & BDA & 1142.9 & 561.9 & 625.5 & 2116 & 0.0 & 1188.9 & 552.4 & 1763.1 \\
\hline \multirow[t]{3}{*}{$11 \mathrm{i}$} & \multirow[t]{3}{*}{5100} & JM & 2725.4 & 1002.6 & 1113.0 & 2077 & 305.9 & 2082.1 & 958.7 & 3186.7 \\
\hline & & DA & 2724.5 & 1015.5 & 1130.6 & 2116 & 305.9 & 2148.9 & 998.3 & 3186.7 \\
\hline & & BDA & 1676.7 & 561.9 & 625.5 & 2116 & 0.0 & 1188.9 & 552.4 & 1763.1 \\
\hline
\end{tabular}

Table D9

Solutions of long-term scenario for case 12 .

\begin{tabular}{|c|c|c|c|c|c|c|c|c|c|c|}
\hline $\begin{array}{l}\text { Case } \\
\mathrm{Nb} .\end{array}$ & $\begin{array}{l}\beta \\
(\$)\end{array}$ & Mechanism & $\begin{array}{l}\text { Objective } \\
\left(\$ \times 10^{6}\right)\end{array}$ & $\begin{array}{l}\text { Exp. sales } \\
\left(\times 10^{3} \text { vehicles }\right)\end{array}$ & $\begin{array}{l}Q \\
\left(\times 10^{3} \text { vehicles }\right)\end{array}$ & $\begin{array}{l}r \\
(\$)\end{array}$ & $\begin{array}{l}B_{d} \\
\left(\$ \times 10^{6}\right)\end{array}$ & $\begin{array}{l}B_{r} \\
\left(\$ \times 10^{6}\right)\end{array}$ & $\begin{array}{l}\mathrm{E}[\mathrm{P}(\mathrm{Q})] \\
\left(\$ \times 10^{6}\right)\end{array}$ & $\begin{array}{l}\mu\left(B_{d}\right) \\
\left(\times 10^{3} \text { vehicles }\right)\end{array}$ \\
\hline \multirow[t]{3}{*}{$12 \mathrm{a}$} & \multirow[t]{3}{*}{3200} & JM & 1338.1 & 816.6 & 830.4 & 1299 & 214.5 & 1060.4 & 385.5 & 3018.8 \\
\hline & & DA & 964.4 & 1051.4 & 1095.7 & 2116 & 175.3 & 2224.7 & 1136.9 & 2931.0 \\
\hline & & BDA & 627.6 & 578.9 & 603.4 & 2116 & 0.0 & 1225.0 & 626.0 & 1614.0 \\
\hline \multirow[t]{3}{*}{$12 \mathrm{~b}$} & \multirow[t]{3}{*}{4150} & JM & 2178.1 & 946.9 & 970.2 & 1549 & 285.1 & 1466.5 & 624.4 & 3129.8 \\
\hline & & DA & 1998.9 & 1116.2 & 1163.3 & 2116 & 271.5 & 2361.9 & 1207.0 & 3111.8 \\
\hline & & BDA & 1177.6 & 578.9 & 603.4 & 2116 & 0.0 & 1225.0 & 626.0 & 1614.0 \\
\hline \multirow[t]{3}{*}{$12 c$} & \multirow[t]{3}{*}{5100} & JM & 3130.0 & 1054.0 & 1088.1 & 1799 & 349.3 & 1896.3 & 891.7 & 3199.1 \\
\hline & & DA & 3075.0 & 1146.4 & 1194.8 & 2116 & 345.9 & 2425.8 & 1239.7 & 3196.0 \\
\hline & & BDA & 1727.6 & 578.9 & 603.4 & 2116 & 0.0 & 1225.0 & 626.0 & 1614.0 \\
\hline \multirow[t]{3}{*}{$12 \mathrm{~d}$} & \multirow[t]{3}{*}{3200} & JM & 996.8 & 633.6 & 647.8 & 1388 & 151.6 & 879.2 & 327.1 & 2980.9 \\
\hline & & DA & 762.9 & 819.5 & 858.8 & 2116 & 125.4 & 1734.0 & 846.7 & 2904.6 \\
\hline & & BDA & 592.2 & 546.3 & 572.5 & 2116 & 0.0 & 1156.0 & 564.5 & 1936.3 \\
\hline \multirow[t]{3}{*}{$12 \mathrm{e}$} & \multirow[t]{3}{*}{4150} & JM & 1658.0 & 754.2 & 778.6 & 1672 & 211.2 & 1260.9 & 542.1 & 3104.7 \\
\hline & & DA & 1570.6 & 872.6 & 914.5 & 2116 & 204.2 & 1846.4 & 901.6 & 3092.8 \\
\hline & & BDA & 1111.2 & 546.3 & 572.5 & 2116 & 0.0 & 1156.0 & 564.5 & 1936.3 \\
\hline \multirow[t]{3}{*}{$12 \mathrm{f}$} & \multirow[t]{3}{*}{5100} & JM & 2423.7 & 855.3 & 891.5 & 1955 & 265.8 & 1672.5 & 786.6 & 3181.3 \\
\hline & & DA & 2412.5 & 897.3 & 940.4 & 2116 & 265.1 & 1898.7 & 927.1 & 3180.4 \\
\hline & & BDA & 1630.2 & 546.3 & 572.5 & 2116 & 0.0 & 1156.0 & 564.5 & 1936.3 \\
\hline \multirow[t]{3}{*}{$12 \mathrm{~g}$} & \multirow[t]{3}{*}{3200} & JM & 788.9 & 508.7 & 522.6 & 1459 & 96.8 & 742.1 & 278.2 & 2974.0 \\
\hline & & DA & 634.0 & 658.1 & 692.7 & 2116 & 79.4 & 1392.5 & 654.5 & 2909.6 \\
\hline & & BDA & 566.3 & 522.4 & 550.0 & 2116 & 0.0 & 1105.5 & 519.6 & 2309.9 \\
\hline \multirow[t]{3}{*}{$12 \mathrm{~h}$} & \multirow[t]{3}{*}{4150} & JM & 1325.7 & 618.2 & 642.5 & 1770 & 145.5 & 1094.3 & 469.6 & 3103.8 \\
\hline & & DA & 1282.4 & 700.3 & 737.2 & 2116 & 142.0 & 1481.9 & 696.5 & 3096.4 \\
\hline & & BDA & 1062.6 & 522.4 & 550.0 & 2116 & 0.0 & 1105.5 & 519.6 & 2309.9 \\
\hline \multirow[t]{3}{*}{$12 \mathrm{i}$} & \multirow[t]{3}{*}{5100} & JM & 1958.4 & 711.7 & 748.2 & 2080 & 190.5 & 1480.6 & 690.6 & 3183.4 \\
\hline & & DA & 1957.9 & 720.0 & 757.9 & 2116 & 190.5 & 1523.5 & 716.1 & 3183.4 \\
\hline & & BDA & 1559.0 & 522.4 & 550.0 & 2116 & 0.0 & 1105.5 & 519.6 & 2309.9 \\
\hline
\end{tabular}




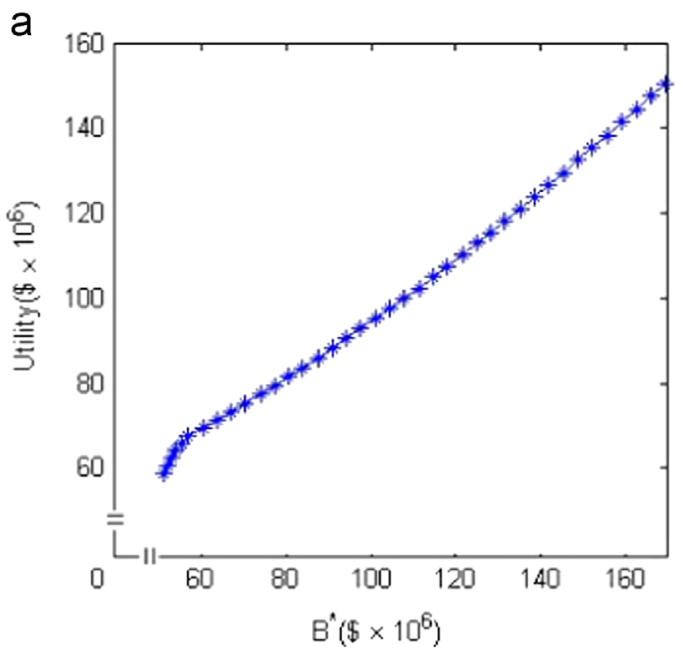

Optimal budget vs utility

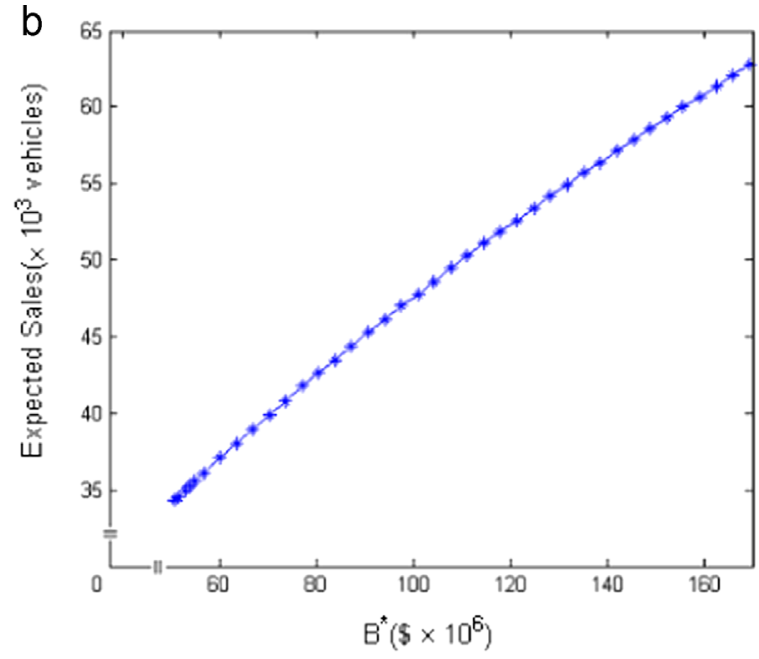

Optimal budget vs expected sales

Fig. 1. Examples of Pareto optimal curves.

\section{Appendix E. Examples for Pareto optimal curves}

We present illustrative examples of Pareto optimal curves based on the solutions of one case with lognormal distribution, $c v=1.2$, and $s=\$ 23,040$. Figures plot the utility and expected sales values in the increasing order of $\beta$ s. Note that each star corresponds to a different $\beta$ value with its budget amount on the horizontal axis. If one solves the subproblem provided in Appendix $C$ with the reported budget values, the same solutions will be obtained.

Fig. 1a shows the optimal budget amounts and the resulting utility values as defined in (15). Fig. 1b illustrates optimal budget amounts and expected sales. As expected, expected sales shows a diminishing rate of increase with respect to increase in budget.

\section{References}

[1] Arcelus Francisco J, Kumar Satyendra, Srinivasan Gopalan. Pricing, rebate, advertising and ordering policies of a retailer facing price-dependent stochastic demand in newsvendor framework under different risk preferences. International Transactions in Operational Research 2006;13(3):209-27.

[2] Arifoğlu Kenan, Deo Sarang, Iravani Seyed MR. Consumption externality and yield uncertainty in the influenza vaccine supply chain: interventions in demand and supply sides. Management Science 2012;58(6):1072-91.

[3] Bard Jonathan F. Practical bilevel optimization: algorithms and applications. Dordrecht, The Netherlands: Kluwer Academic Publishers; 1998.

[4] Bard Jonathan F, Plummer John, Sourie Jean Claude. A bilevel programming approach to determining tax credits for biofuel production. European Journal of Operational Research 2000;120(1):30-46.

[5] Gerard Cachon P. Supply chain coordination with contracts. In: Handbooks in operations research and management science, vol. 11; 2003. p. 227-339.

[6] California Energy Commission. California energy demand forecast 2012-2022 volume 1: statewide electricity demand and methods, end-user natural gas demand, and energy efficiency. Technical Report CEC2002012001SFVI, California Energy Commission; June 2012.

[7] California Environmental Protection Agency. California, clean vehicle rebate project; 2014. URL 〈https://cleanvehiclerebate.org/eng〉.

[8] Chick Stephen E, Mamani Hamed, Simchi-Levi David. Supply chain coordination and influenza vaccination. Operations Research 2008;56(6):1493-506.

[9] Cohen Maxime C, Lobel Ruben, Perakis Georgia. The impact of demand uncertainty on consumer subsidies for green technology adoption. Management Science 2016;62(5):1235-58.

[10] Colson Benoît, Marcotte Patrice, Savard Gilles. An overview of bilevel optimization. Annals of Operations Research 2007;153(1):235-56.

[11] Cesare Luigi De, Liddo Andrea Di. A stackelberg game of innovation diffusion: pricing, advertising and subsidy strategies. International Game Theory Review 2001;3(4):325-39.

[12] Deo Sarang, Corbett Charles J. Cournot competition under yield uncertainty: the case of the US influenza vaccine market. Manufacturing and Service Operations Management 2009;11(4):563-76.
[13] Eberle Ulrich, Helmolt Rittmar von. Sustainable transportation based on electric vehicle concepts: a brief overview. Energy and Environmental Science 2010;3(6):689-99.

[14] Horsky Dan, Simon Leonard S. Advertising and the diffusion of new products. Marketing Science 1983;2(1):1-17.

[15] Huang Jian, Leng Mingming, Liang Liping, Liu Jian. Promoting electric automobiles: supply chain analysis under a government's subsidy incentive scheme. IIE Transactions 2013;45(8):826-44.

[16] Jones Chris. Applied welfare economics. New York: Oxford; 2005.

[17] Joskow Paul L, Rose Nancy L. The effects of economic regulation. In: Handbook of industrial organization; 1989. p. 1449-1506.

[18] Kalish Shlomo, Lilien Gary L. Optimal price subsidy policy for accelerating the diffusion of innovation. Marketing Science 1983;2(4):407-20.

[19] Khouja Moutaz, Robbins Stephanie S. Linking advertising and quantity decisions in the single-period inventory model. International Journal of Production Economics 2003;86(2):93-105.

[20] Kim Jerim, Son Sung-Yong, Lee Jung-Min, Ha Hyung-Tae. Scheduling and performance analysis under a stochastic model for electric vehicle charging stations. Omega 2016.

21] Krass Dmitry, Nedorezov Timur, Ovchinnikov Anton. Environmental taxes an the choice of green technology. Production and Operations Management 2013;22(5):1035-55.

22] Lee Chih-Ming, Hsu Shu-Lu. The effect of advertising on the distribution-free newsboy problem. International Journal of Production Economics 2011;129 (1):217-24.

23] Li Susan X, Huang Zhimin, Zhu Joe, Chau Patrick YK. Cooperative advertising game theory and manufacturer-retailer supply chains. Omega 2002;30 (5):347-57.

[24] Liu Huihui, Lei Ming, Deng Honghui, Keong Leong G, Huang Tao. A dual channel, quality-based price competition model for the WEEE recycling market with government subsidy. Omega 2016;59:290-302.

[25] Lobel Ruben, Perakis Georgia. Consumer choice model for forecasting demand and designing incentives for solar technology. Working Paper, Massachusetts Institute of Technology, Cambridge, MA; 2011.

[26] Lu Wen-Min, Kweh Qian Long, Nourani Mohammad, Huang Feng-Wen. Evaluating the efficiency of dual-use technology development programs from the R\&D and socio-economic perspectives. Omega 2016;62:82-92.

[27] Mamani Hamed, Adida Elodie, Dey Debabrata. Vaccine market coordination using subsidy. IIE Transactions on Healthcare Systems Engineering 2012;2 (1):78-96.

[28] Nissan Motor Corporation. Nissan leaf global sales reach 100,000 units. URL 〈http://www.nissan-global.com/EN/NEWS/〉; September 2014.

[29] Nissan USA. Nissan cars, trucks, crossovers, \& SUVs-Nissan USA. URL 〈http:// www.nissanusa.com〉; September 2013.

[30] Raz Gal, Ovchinnikov Anton. Coordinating pricing and supply of public interest goods using government rebates and subsidies. IEEE Transactions on Engineering Management 2015;62(1):65-79.

[31] Schneeweiß Christoph. Hierarchical structures in organisations: a conceptual framework. European Journal of Operational Research 1995;86(1):4-31.

[32] Schneeweiss Christoph. Distributed decision making-a unified approach. European Journal of Operational Research 2003;150(2):237-52.

[33] Taylor Terry A, Xiao Wenqiang. Subsidizing the distribution channel: dono funding to improve the availability of malaria drugs. Management Science 2014;60(10):2461-77.

[34] Train Kenneth E. Optimal regulation: the economic theory of natural monopoly. Cambridge, MIT Press Books; 1991. 
[35] True Car Inc. 〈http://www.truecar.com/prices-new/nissan/leaf-pricing/〉; September 2013.

[36] US Department of Energy. One million electric vehicles by 2015, February 2011 status report. 〈http://www1.eere.energy.gov/vehiclesandfuels/pdfs/1_million_ electric_vehicles_rpt.pdf); September 2011.

[37] US Department of Energy. Alternative fuels data center: hybrid and plug-in electric vehicles. 〈http://www.afdc.energy.gov/vehicles/electric.html〉; September 2014.

[38] WBCSD. Mobility 2030: meeting the challenges to sustainability. World Business Council for Sustainable Development; 2004.
[39] Wernz Christian, Deshmukh Abhijit. Multiscale decision-making: bridging organizational scales in systems with distributed decision-makers. European Journal of Operational Research 2010;202(3):828-40.

[40] Zhang Hui, Wernz Christian, Slonim Anthony D. Aligning incentives in health care: a multiscale decision theory approach. EURO Journal on Decision Processes 2015:1-26. 